\title{
Algebras versus coalgebras
}

\author{
Robert Wisbauer \\ University of Düsseldorf, Germany \\ e-mail: wisbauer@math.uni-duesseldorf.de
}

April 10, 2007

\begin{abstract}
Algebras and coalgebras are fundamental notions for large parts of mathematics. The basic constructions from universal algebra are now expressed in the language of categories and thus are accessible to classical algebraists and topologists as well as to logicians and computer scientists. Some of them have developed specialised parts of the theory and often reinvented constructions already known in a neighbouring area. One purpose of this survey is to show the connection between results from different fields and to trace a number of them back to some fundamental papers in category theory from the early 70's.

Another intention is to look at the interplay between algebraic and coalgebraic notions. Hopf algebras are one of the most interesting objects in this setting. While knowledge of algebras and coalgebras are folklore in general category theory, the notion of Hopf algebras is usually only considered for monoidal categories. In the course of the text we do suggest how to overcome this defect by defining a Hopf monad on an arbitrary category as a monad and comonad satisfying some compatibility conditions and inducing an equivalence between the base category and the category of the associated bimodules. For a set $G$, the endofunctor $G \times$ - on the category of sets shares these properties if and only if $G$ admits a group structure.

Finally, we report about the possibility of subsuming algebras and coalgebras in the notion of $(F, G)$-dimodules associated to two functors $F, G: \mathbb{A} \rightarrow \mathbb{B}$ between different categories. This observation, due to Tatsuya Hagino, was an outcome from the theory of categorical data types and may also be of use in classical algebra.
\end{abstract}

Contents: 1.Introduction, 2.Modules and comodules, 3.Relations between functors, 4.Relations between endofunctors, 5.Combining monads and comonads, 6. $(F, G)$-dimodules. 


\section{Introduction}

The purpose of Universal Algebra is to develop a general theory which applies to a large part of algebraic structures. Initially it started with the study of abstract algebras, that is, sets $A$ with a collection of ( $n$-ary) operations on $A$. In his thesis $(1963$, [32]) Lawvere suggested to formulate these general settings in the language of categories and functors.

In particular it turned out that adjoint pairs of functors are of central importance. The study of monads (triples) and their modules and comonads (cotriples) and their comodules was initiated by Eilenberg and Moore in [21] and in Beck [5]. This approach covers an extremely wide range of applications including not only the classical notions from module theory and topology but also those from model theory and logic, the latter being of growing interest for computer science.

Bringing the notions down to the language of categories and functors, the common part of all theories envisaged has to be expressed in this terminology. Many interesting observations in these areas can be traced back and understood with the abstract handling of functors as suggested by Eilenberg, Mac Lane and their schools. To make this clear we will pay some attention to the so called mixed distributive laws between endofunctors introduced in Beck [6] and further studied in van Osdol [54] and Wolff [57] (see also Barr and Wells [4] and Škoda [46, Section 9]). These were rediscovered in Turi and Plotkin [52] in the context of operational semantics and by Brzeziński and Majid [14] in the form of entwining structures between the tensor product of an algebra and a coalgebra over a commutative ring $R$. Some of these notions were more generally handled in monoidal categories by Mesablishvili in [40]. The action of monoidal categories on any category as considered by Škoda in [45] puts this in a wider context.

We note that for some purposes it is natural to consider monads and comonads in 2-categories and we refer to Street [47], Lack and Street [31], Power and Watanabe [43], Lenisa, Power and Watanabe [33] and Tanaka and Power [51] for a treatment in this direction. Szlachányi also considers 2-categories in [48] to understand (op)monoidal functors for bialgebroids. His approach is similar in spirit to our investigations but his focus is on different results.

Now each field of application has its own requirements and the questions and constructions arising, say, in computer science may also be of relevance for classical modules. In fact this lecture was motivated by the observation that manipulations suggested and performed by computer scientists (e.g. [52]) are also of interest for corings and comodules.

Dualising the algebraic theory yields the coalgebraic theory and both of these have their realms of applications. However, there are also combinations 
of constructions from both of them and one purpose of this presentation is to outline how this can be done and what the outcome is.

As some other authors do (e.g. [11], [45]) we modify the terminology from Eilenberg and Moore in [21] by referring to the F-algebras of an endofunctor in their sense as F-modules, and F-coalgebras as F-comodules. This is in total correspondence to the application in module theory where the functor $F$ plays the role of an algebra or coalgebra, respectively, while the attached categories are modules or comodules. We hope this change does not lead to conflicts in other situations.

At the core of our treatise is Johnstone's general Lifting of functors theorem 3.1 from which many applications can be understood and derived. A different handling of related problems can be found in Mesablishvili [39] where a generalisation of descent theory is proposed.

As a byproduct, on our way we obtain some of the conditions on a monad $S$ on a monoidal category $\mathcal{C}$ from Moerdijk [41, Proposition 1.4] which allow the tensor product to lift from $\mathcal{C}$ to the category of $S$-modules (see 3.4). Monads of this type were named Hopf monads in [41], called opmonoidal monad in McCrudden [37, Example 2.5] and Szachlányi [48, Definition 2.4], and bimonads in Bruguières and Virelizier [11, 2.3]. We do not use these notions but will associate a different meaning to the term Hopf monad here.

Classical Hopf algebras $H$ over a commutative ring $R$ have compatible algebra and coalgebra structures $H \otimes_{R} H \rightarrow H$ and $H \rightarrow H \otimes_{R} H$ such that the free functor $\phi^{H}: R$-Mod $\rightarrow R$-Mod ${ }_{H}^{H}$ induces a category equivalence (e.g. $[16,15.5])$. Traditionally most generalisations of Hopf algebras are based on a kind of tensor product on a category which allows us to follow the usual arguments at least for a larger part. Trying to have the aforementioned equivalence in utmost generality for an endofunctor $B$ on an arbitrary category $\mathbb{A}$, one has to impose all conditions necessary on the functor $B$. Thus we require $B$ to be a monad and a comonad whose compatibility is controlled by a natural transformation $\lambda: B B \rightarrow B B$ (mixed distributive law). We call this a (mixed) bimonad and suggest naming it a Hopf monad provided the related free functor $\phi_{B}^{B}: \mathbb{A} \rightarrow \mathbb{A}_{B}^{B}$ induces an equivalence (see 5.15). In case $\lambda$ is derived from a natural transformation $\tau: B B \rightarrow B B$ which satisfies the Yang-Baxter equation, following Takeuchi, $B$ may be called a braided bimonad or braided Hopf monad if $B$ is a Hopf monad (see 5.17). We note that natural transformations satisfying the Yang-Baxter equation are also used by Menini and Stefan in [38] to define compatible flip morphisms for monads on arbitrary categories and their concept was extended by Kasangian, Lack and Vitale in [29] (see 5.18). By a suggestion of Manin, in noncommutative geometry the role of the twist map can be replaced by an arbitrary YangBaxter operator (on vector space categories). This approach leads to similar formulas and is developed by Baez in [2]. Relations between Yang-Baxter op- 
erators and entwining structures (on vector space categories) are investigated by Brzeziński and Nichita in [15].

As a special case we can look at a set $G$ and the endofunctor $G \times-$ on the category of sets. As outlined in Caenepeel and Lombaerde [19], the existence of an antipode for the related bimonad is then equivalent to $G$ having a group structure and implies the fundamental theorem. The notions introduced here deepens the understanding of this situation and in this case the fundamental theorem implies in turn the existence of an antipode (see 5.19, 5.20).

Inspired by questions arising in computer science, Hagino introduced in [25] for two functors $F, G: \mathbb{A} \rightarrow \mathbb{B}$ between arbitrary categories the notion of $(F, G)$-algebras (we call them $(F, G)$-dimodules). These generalise modules as well as comodules of endofunctors and in Section 6 we give a short outline how to treat them in abstract category theory. Their use in (classical) algebra still awaits exploration. For a deeper presentation of examples and usage of (co)algebras in universal algebra and computer science the interested reader may consult Gumm's articles [23, 24].

To avoid confusion, we mention that, as usual, functor symbols are written on the left side of an object. When referring to examples in module categories over a commutative ring $R$, following the usage of our main references, we usually consider right tensor functors $-\otimes X, X$ an $R$-module. The reader should be aware that this causes a reversion of symbols in some of the diagrams or formulas when passing from arbitrary categories to $R$-modules.

\section{Modules and comodules}

To fix notation let us recall some basic facts.

2.1. Categories. A category $\mathbb{A}$ consists of a class of objects $\operatorname{Obj}(\mathbb{A})$ and a class of morphism sets $\operatorname{Mor}(\mathbb{A})$ satisfying

(i) for objects $A, B$ in $\mathbb{A}$ there is a morphism set $\operatorname{Mor}_{\mathbb{A}}(A, B)$ such that

$$
\operatorname{Mor}_{\mathbb{A}}(A, B) \cap \operatorname{Mor}_{\mathbb{A}}\left(A^{\prime}, B^{\prime}\right)=\emptyset \text { for }(A, B) \neq\left(A^{\prime}, B^{\prime}\right) ;
$$

(ii) for $A, B, C \in \operatorname{Obj}(\mathbb{A})$ there is a composition map

$$
\operatorname{Mor}_{\mathbb{A}}(A, B) \times \operatorname{Mor}_{\mathbb{A}}(B, C) \rightarrow \operatorname{Mor}_{\mathbb{A}}(A, C),(f, g) \mapsto g f ;
$$

(iii) for every $A \in \operatorname{Obj}(\mathbb{A})$ there is an identity morphism $I_{A} \in \operatorname{Mor}_{\mathbb{A}}(A, A)$.

The connection between two categories is given by

2.2. Functors. A covariant functor $F: \mathbb{A} \rightarrow \mathbb{B}$ between two categories consists of assignments

$$
\begin{aligned}
\operatorname{Obj}(\mathbb{A}) & \rightarrow \operatorname{Obj}(\mathbb{B}), \quad A \\
\operatorname{Mor}(\mathbb{A}) & \rightarrow \operatorname{Mor}(\mathbb{B}), \quad f: A \rightarrow B(A),
\end{aligned}
$$


such that $F\left(I_{A}\right)=I_{F(A)}$ and $F(f g)=F(f) F(g)$.

Contravariant functors reverse the composition of morphisms. Here all our functors considered will be covariant.

The relation between two functors is described by

2.3. Natural transformations. Let $F, F^{\prime}: \mathbb{A} \rightarrow \mathbb{B}$ be covariant functors.

A natural transformation $\alpha: F \rightarrow F^{\prime}$ is given by morphisms

$$
\alpha_{A}: F(A) \rightarrow F^{\prime}(A) \text { in } \mathbb{B}, A \in \operatorname{Obj}(\mathbb{A}),
$$

such that $f: A \rightarrow B$ in $\mathbb{A}$ induces the commutative diagram in $\mathbb{B}$

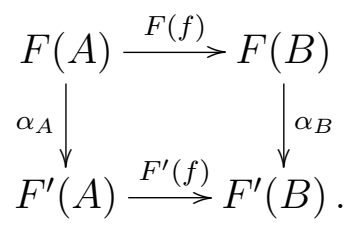

Given another pair of functors $G, G^{\prime}: \mathbb{B} \rightarrow \mathbb{C}$ with natural transformation $\beta: G \rightarrow G^{\prime}$, the diagram

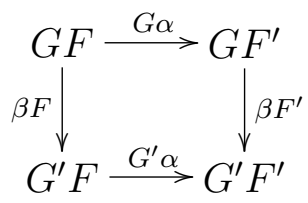

is commutative and thus there is a natural transformation (Godement product)

$$
\beta \alpha:=\beta F^{\prime} \circ G \alpha=G^{\prime} \alpha \circ \beta F: G F \rightarrow G^{\prime} F^{\prime} .
$$

If $\mathbb{A}$ is a small category then the endofunctors (as objects) together with natural transformations form a strict monoidal category: the product of endofunctors is the composition and the composition of natural transformations is given by the Godement product (e.g. [46, Section 8]).

In what follows we will use functors and natural transformations as basic tools for general constructions.

2.4. Adjoint pairs of functors. A pair $(L, R)$ of functors $L: \mathbb{A} \rightarrow \mathbb{B}$ and $R: \mathbb{B} \rightarrow \mathbb{A}$ between categories $\mathbb{A}$ and $\mathbb{B}$ is called adjoint if there are natural isomorphisms (in $A \in \operatorname{Obj}(\mathbb{A})$ and $B \in \operatorname{Obj}(\mathbb{B})$ )

$$
\vartheta_{A, B}: \operatorname{Mor}_{\mathbb{B}}(L(A), B) \rightarrow \operatorname{Mor}_{\mathbb{A}}(A, R(B)) .
$$

Associated to such a pair there are natural transformations

$$
\text { unit } \eta: I_{\mathbb{A}} \rightarrow R L \text { and counit } \varepsilon: L R \rightarrow I_{\mathbb{B}} \text {. }
$$


2.5. $\boldsymbol{F}$-modules. Given an endofunctor $F: \mathbb{A} \rightarrow \mathbb{A}$, an $F$-module $\left(A, \varrho_{A}\right)$ is an $A \in \operatorname{Obj}(\mathbb{A})$ with a morphism in $\mathbb{A}$,

$$
\varrho_{A}: F(A) \rightarrow A .
$$

A morphism $f: A \rightarrow A^{\prime}$ in $\mathbb{A}$ between $F$-modules is an $F$-module morphism provided it induces a commutative diagram

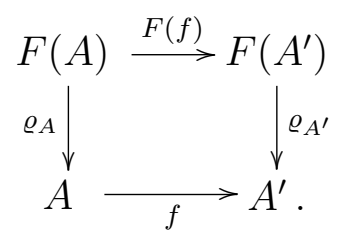

With these morphisms, the $F$-modules form a category which is denoted by $\mathbb{A}_{F}$. There is the faithful forgetful functor

$$
U_{F}: \mathbb{A}_{F} \rightarrow \mathbb{A}, \quad\left(A, \varrho_{A}\right) \mapsto A .
$$

In all generality some limits in $\mathbb{A}_{F}$ are induced from limits in $\mathbb{A}$.

2.6. Proposition. Let $L: \boldsymbol{\Lambda} \rightarrow \mathbb{A}_{F}$ be any functor and $\boldsymbol{\Lambda}$ a small category.

(1) If $\lim _{L} L$ exists in $\mathbb{A}$, then it belongs to $\mathbb{A}_{F}$.

(2) Assume that $F$ preserves colimits. If $\lim _{\longrightarrow} L$ exists in $\mathbb{A}$, then it belongs to $\mathbb{A}_{F}$.

The relations between $\mathbb{A}_{F}$ and $\mathbb{A}$ are even stronger if additional conditions are imposed on the endofunctor $F$.

2.7. Monads. A monad on $\mathbb{A}$ is a triple $\mathbb{F}=(F, \mu, \eta)$, where $F: \mathbb{A} \rightarrow \mathbb{A}$ is a functor and

$$
\mu: F F \rightarrow F, \quad \eta: I_{\mathbb{A}} \rightarrow F,
$$

are natural transformations with commutative diagrams

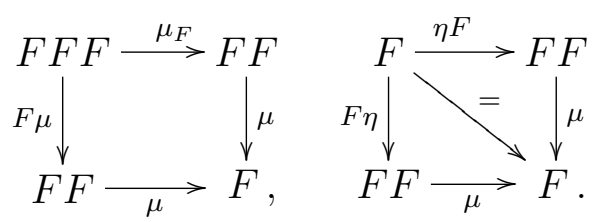

Given two monads $\mathbb{F}=(F, \mu, \eta)$ and $\mathbb{F}^{\prime}=\left(F^{\prime}, \mu^{\prime}, \eta^{\prime}\right)$ on $\mathbb{A}$, a natural transformation $\alpha: F \rightarrow F^{\prime}$ is called a morphism of monads if the following induced diagrams commute:
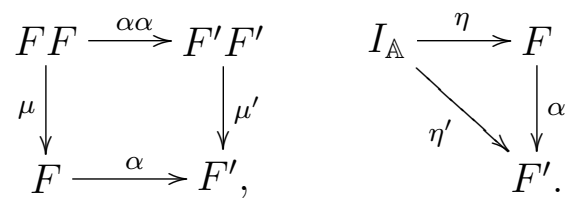
2.8. Monads and their modules. Given a monad $\mathbb{F}=(F, \mu, \eta)$ on $\mathbb{A}$, an $\mathbb{F}$ module is an $A \in \operatorname{Obj}(\mathbb{A})$ and a morphism $\varrho_{A}: F(A) \rightarrow A$ with commutative diagrams
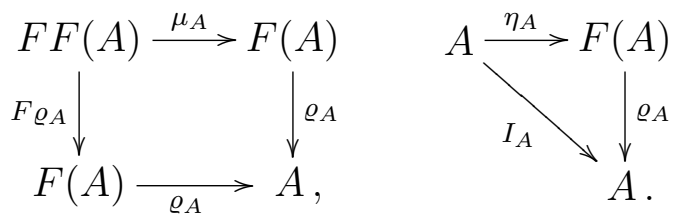

As shown in Eilenberg-Moore [21], for a monad $F$, the forgetful functor $U_{F}: \mathbb{A}_{\mathbb{F}} \rightarrow \mathbb{A}$ is right adjoint to the (free) functor

$$
\begin{aligned}
& \phi_{F}: \mathbb{A} \rightarrow \mathbb{A}_{F}, \quad A \mapsto\left(F(A), F F(A) \stackrel{\mu_{A}}{\longrightarrow} F(A)\right), \\
& A \stackrel{f}{\rightarrow} A^{\prime} \mapsto F(A) \stackrel{F(f)}{\longrightarrow} F\left(A^{\prime}\right),
\end{aligned}
$$

by the isomorphisms for $A \in \operatorname{Obj}(\mathbb{A})$ and $B \in \operatorname{Obj}\left(\mathbb{A}_{\mathbb{F}}\right)$,

$$
\operatorname{Mor}_{\mathbb{A}_{F}}(F(A), B) \rightarrow \operatorname{Mor}_{\mathbb{A}}\left(A, U_{F}(B)\right), \quad f \mapsto f \circ \eta_{A} .
$$

Notice that $U_{F} \phi_{F}=F$.

Dual to the preceding notions there is a theory of comodules which we sketch in the next paragraphs.

2.9. $G$-comodules. For a functor $G: \mathbb{A} \rightarrow \mathbb{A}$, a $G$-comodule $\left(A, \varrho^{A}\right)$ is an $A \in \operatorname{Obj}(\mathbb{A})$ with a morphism in $\mathbb{A}$,

$$
\varrho^{A}: A \rightarrow G(A) .
$$

A morphism $f: A \rightarrow A^{\prime}$ in $\mathbb{A}$ between $G$-comodules is a $G$-comodule morphism inducing the commutative diagram

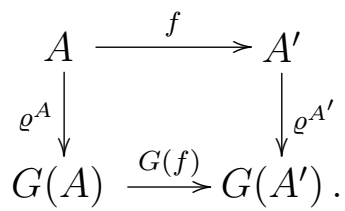

The $G$-comodules together with $G$-comodule morphisms form a category which we denote by $\mathbb{A}^{G}$. The forgetful functor is faithful,

$$
U^{G}: \mathbb{A}^{G} \rightarrow \mathbb{A}, \quad\left(A, \varrho^{A}\right) \mapsto A .
$$

As a sample of the relation between the categories $\mathbb{A}$ and $\mathbb{A}^{G}$ we mention:

2.10. Proposition. Let $L: \Lambda \rightarrow \mathbb{A}^{G}$ be any functor and $\boldsymbol{\Lambda}$ a small category.

(1) If $\lim _{\longrightarrow} L$ exists in $\mathbb{A}$, then it belongs to $\mathbb{A}^{G}$. 
(2) Assume that $G$ preserves limits. If $\lim _{\longleftarrow} L$ exists in $\mathbb{A}$, then it belongs to $\mathbb{A}^{G}$.

For more information about the behaviour of limits and colimits and examples of modules and comodules in the category of sets we refer to Adámek and Porst [1].

2.11. Comonads. A comonad is a triple $\mathbb{G}=(G, \delta, \varepsilon)$, where $G: \mathbb{A} \rightarrow \mathbb{A}$ is a functor and

$$
\delta: G \rightarrow G G, \quad \varepsilon: G \rightarrow I_{\mathbb{A}},
$$

are natural transformations with commuting diagrams
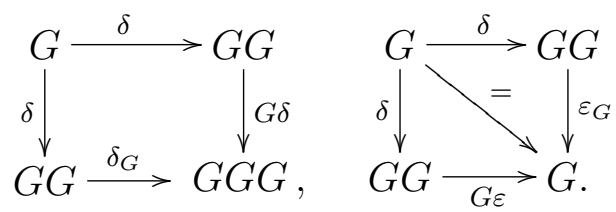

Given two comonads $\mathbb{G}=(G, \delta, \varepsilon)$ and $\mathbb{G}^{\prime}=\left(G^{\prime}, \delta^{\prime}, \varepsilon^{\prime}\right)$, a natural transformation $\beta: G \rightarrow G^{\prime}$ is called a morphism of comonads if the following diagrams commute:
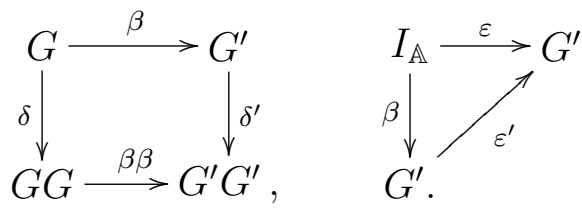

2.12. Comonads and their comodules. Let $\mathbb{G}=(G, \delta, \varepsilon)$ be a comonad. A $\mathbb{G}$-comodule is an object $A \in \operatorname{Obj}(\mathbb{A})$ with a morphism

$$
\varrho^{A}: A \rightarrow G(A) \text { in } \mathbb{A}
$$

inducing commutative diagrams
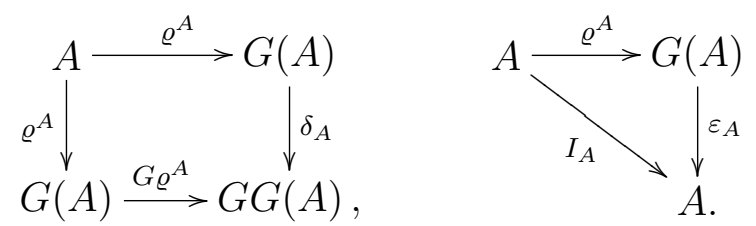

The forgetful functor $U^{G}: \mathbb{A}^{G} \rightarrow \mathbb{A}$ is left adjoint to the (free) functor

$$
\begin{aligned}
& \phi^{G}: \mathbb{A} \rightarrow \mathbb{A}^{G}, \quad A \mapsto\left(G(A), G(A) \stackrel{\delta_{A}}{\longrightarrow} G G(A)\right), \\
& A \stackrel{f}{\rightarrow} A^{\prime} \mapsto G(A) \stackrel{G(f)}{\longrightarrow} G\left(A^{\prime}\right),
\end{aligned}
$$


by the isomorphisms

$$
\operatorname{Mor}_{\mathbb{A} G}(B, G(A)) \rightarrow \operatorname{Mor}_{\mathbb{A}}\left(U^{G}(B), A\right), \quad f \mapsto \varepsilon_{A} \circ f,
$$

for any $A \in \operatorname{Obj}(\mathbb{A})$ and $B \in \operatorname{Obj}\left(\mathbb{A}^{G}\right)$. Notice that $U^{G} \phi^{G}=G$.

We now recall that monads and comonads are closely related to adjoint pairs of functors (e.g. [21]):

2.13. Adjoint pairs and (co)monads. Let $L: \mathbb{A} \rightarrow \mathbb{B}$ and $R: \mathbb{B} \rightarrow \mathbb{A}$ be an adjoint pair of functors (see 2.4) with

$$
\text { unit } \eta: I_{\mathbb{A}} \rightarrow R L \text { and counit } \varepsilon: L R \rightarrow I_{\mathbb{B}},
$$

Then $R L: \mathbb{A} \rightarrow \mathbb{A}$ has a monad structure with

$$
\text { product } \mu=R \varepsilon_{L}: R L R L \rightarrow R L \text { and unit } \eta: I_{\mathbb{A}} \rightarrow R L,
$$

and $L R: \mathbb{B} \rightarrow \mathbb{B}$ has a comonad structure with

$$
\text { coproduct } \delta=L \eta_{R}: L R \rightarrow L R L R \text { and counit } \varepsilon: L R \rightarrow I_{\mathbb{B}} .
$$

\section{Relations between functors}

To study the relationship between various module categories, the following definition is of interest. It was formulated in Johnstone [27] for monads but we also consider it for arbitrary endofunctors.

3.1. Lifting of functors. Let $F$ and $G$ be endofunctors of the categories $\mathbb{A}$ and $\mathbb{B}$ respectively. Given functors

$$
T: \mathbb{A} \rightarrow \mathbb{B}, \quad \bar{T}: \mathbb{A}_{F} \rightarrow \mathbb{B}_{G}, \text { and } \widehat{T}: \mathbb{A}^{F} \rightarrow \mathbb{B}^{G}
$$

we say that $\bar{T}$ (resp. $\widehat{T}$ ) is a lifting of $T$ provided the left (resp. right) diagram
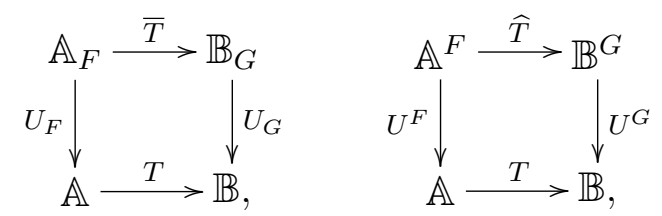

is commutative, where the U's denote the forgetful functors.

The following assertions are easy to verify.

3.2. Proposition. With the notation in 3.1, consider the functors

$$
T F, G T: \mathbb{A} \rightarrow \mathbb{B}
$$


(1) For any natural transformation $\lambda: G T \rightarrow T F$, the functor

$$
\begin{aligned}
\bar{T}: \mathbb{A}_{F} \rightarrow \mathbb{B}_{G}, \quad\left(A, \varrho_{A}\right) & \mapsto\left(T(A), T\left(\varrho_{A}\right) \circ \lambda_{A}\right), \\
A \stackrel{f}{\longrightarrow} A^{\prime} & \mapsto T(A) \stackrel{T(f)}{\longrightarrow} T\left(A^{\prime}\right),
\end{aligned}
$$

is a lifting of $T: \mathbb{A} \rightarrow \mathbb{B}$.

(2) For any natural transformation $\varphi: T F \rightarrow G T$, the functor

$$
\begin{aligned}
\widehat{T}: \mathbb{A}^{F} \rightarrow \mathbb{B}^{G}, \quad\left(A, \varrho^{A}\right) & \mapsto\left(T(A), \varphi_{A} \circ T\left(\varrho^{A}\right)\right), \\
A \stackrel{f}{\longrightarrow} A^{\prime} & \mapsto T(A) \stackrel{T(f)}{\longrightarrow} T\left(A^{\prime}\right),
\end{aligned}
$$

is a lifting of $T: \mathbb{A} \rightarrow \mathbb{B}$.

For monads and comonads there exist bijections between liftings and certain natural transformations.

3.3. Lifting for monads. (Applegate) Let $\mathbb{F}=(F, \mu, \eta)$ and $\mathbb{G}=\left(G, \mu^{\prime}, \eta^{\prime}\right)$ be monads on the categories $\mathbb{A}$ and $\mathbb{B}$, respectively, and let $T: \mathbb{A} \rightarrow \mathbb{B}$ be a functor.

(1) The liftings $\bar{T}: \mathbb{A}_{\mathbb{F}} \rightarrow \mathbb{B}_{\mathbb{G}}$ of $T$ are in bijective correspondence with the natural transformations $\lambda: G T \rightarrow T F$ inducing commutative diagrams
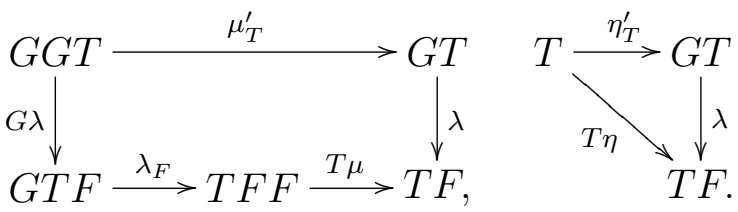

(2) If $\mathbb{A}_{F}$ has coequalisers of reflexive pairs and $T$ has a left adjoint, then any lifting $\bar{T}$ has a left adjoint.

(3) Assume there is a lifting $\bar{T}: \mathbb{A}_{\mathbb{F}} \rightarrow \mathbb{B}_{\mathbb{G}}$ with invertible $\lambda: G T \rightarrow T F$. If $T$ has a right adjoint, then $\bar{T}$ has a right adjoint.

Proof. This is proved in Johnstone [27, Lemma 1, Theorem 4 and 2].

For $\lambda$, the left hand diagram corresponds to $\lambda_{A}$ being a $G$-module morphism for every $F$-module $\left(A, \varrho_{A}\right)$ and the right hand diagram is related to unitality.

Given a lifting $\bar{T}: \mathbb{A}_{\mathbb{F}} \rightarrow \mathbb{B}_{\mathbb{G}}, \lambda$ is obtained in the following way. For any $A \in \operatorname{Obj}(\mathbb{A})$ there is an isomorphism

$$
\alpha_{A}: \operatorname{Mor}_{\mathbb{B}_{G}}\left(\phi_{G} T(A), \bar{T} \phi_{F}(A)\right) \rightarrow \operatorname{Mor}_{\mathbb{B}}\left(T(A), U_{G} \bar{T} \phi_{F}(A)\right) .
$$

Then $T\left(\eta_{A}\right): T(A) \rightarrow T F(A)=T U_{F} \phi_{F}(A)=U_{G} \bar{T} \phi_{F}(A)$ belongs to the right side of the isomorphism and we obtain $\bar{\lambda}(A)=\alpha_{A}^{-1}\left(T\left(\eta_{A}\right)\right): \phi_{G} T(A) \rightarrow$ $\bar{T} \phi_{F}(A)$. This yields

$$
\lambda=U_{G} \bar{\lambda}: G T=U_{G} \phi_{G} T \rightarrow U_{G} \bar{T} \phi_{F}=T U_{F} \phi_{F}=T F .
$$


3.4. Lifting of a tensor product to modules. The above proposition can be applied to characterise monads $(S, \mu, \eta)$ on a monoidal category $(\mathcal{C}, \otimes, E)$ (see [35]) for which the monoidal structure from $\mathcal{C}$ lifts to the category $\mathcal{C}_{S}$ of $S$-modules. The situation is described by the diagram

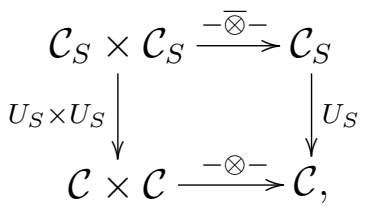

By 3.3(1), for the existence of some $-\bar{\otimes}-$ making the diagram commute one needs a natural transformation

$$
\lambda: S(X \otimes Y) \rightarrow S(X) \otimes S(Y)
$$

yielding commutative diagrams

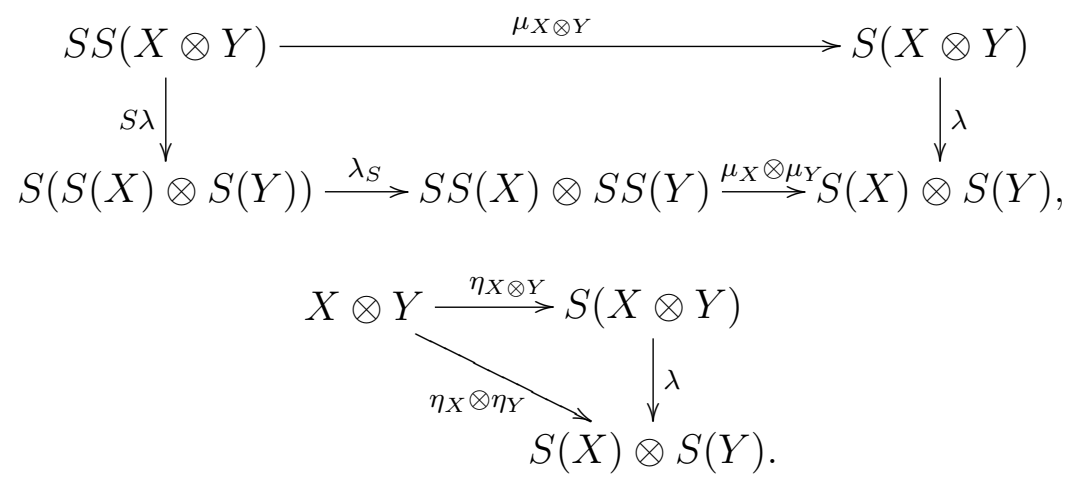

This corresponds to the first three diagrams in [41, Section 7].

To make $\mathcal{C}_{S}$ a unital tensor category one has to require that $E$ is an $S$ module for some morphism $S(E) \rightarrow E$ in $\mathcal{C}$ and that the coherence conditions are respected (diagrams (4) to (7) in [41, Section 7$]$ ). Such monads are called Hopf monads in Moerdijk [41] (see introduction).

3.5. Lifting for comonads. Let $\mathbb{F}=(F, \delta, \varepsilon)$ and $\mathbb{G}=\left(G, \delta^{\prime}, \varepsilon^{\prime}\right)$ be comonads on the categories $\mathbb{A}$ and $\mathbb{B}$, respectively, and let $T: \mathbb{A} \rightarrow \mathbb{B}$ be a functor.

(1) The liftings $\widehat{T}: \mathbb{A}^{\mathbb{F}} \rightarrow \mathbb{B}^{\mathbb{G}}$ of $T$ are in bijective correspondence with the natural transformations $\varphi: T F \rightarrow G T$ inducing commutativity of the diagrams
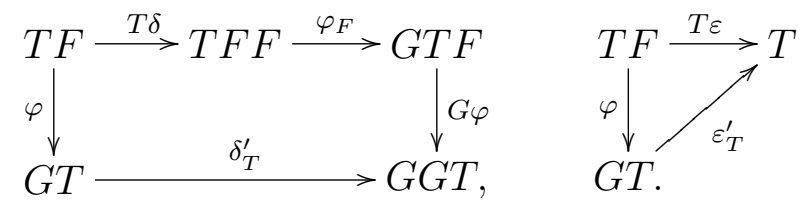
(2) If $\mathbb{A}^{F}$ has equalisers of reflexive pairs and $T$ has a right adjoint, then any lifting $\widehat{T}$ has a right adjoint.

(3) Assume there is a lifting $\widehat{T}: \mathbb{A}^{\mathbb{F}} \rightarrow \mathbb{B}^{\mathbb{G}}$ with $\varphi: T F \rightarrow G T$ invertible. If $T$ has a left adjoint, then the lifting $\widehat{T}$ has a left adjoint.

Proof. Dual to 3.3.

3.6. Lifting of a tensor product to comodules. Let $(T, \delta, \varepsilon)$ be a comonad on a monoidal category $(\mathcal{C}, \otimes, E)$ for which the monoidal structure from $\mathcal{C}$ lifts to the category $\mathcal{C}^{T}$ of $T$-comodules. The situation is described by the diagram

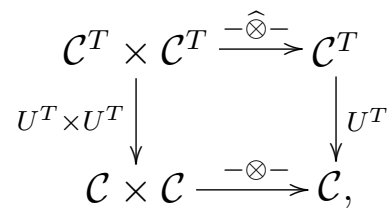

By 3.5(1) for the existence of some $-\widehat{\otimes}-$ making the diagram commute one needs a natural transformation

$$
\varphi: T(X) \otimes T(Y) \rightarrow T(X \otimes Y)
$$

and a morphism $E \rightarrow T(E)$ yielding the commutative diagrams as required by 3.5 plus appropriate conditions to assure the coherence conditions.

\section{Relations between endofunctors}

In this section we will specialise the preceding observations to $\mathbb{A}=\mathbb{B}$ and endofunctors.

4.1. Lifting of the identity. Let $\mathbb{F}=(F, \mu, \eta), \mathbb{F}^{\prime}=\left(F^{\prime}, \mu^{\prime}, \eta^{\prime}\right)$ be monads and $\mathbb{G}=(G, \delta, \varepsilon), \mathbb{G}^{\prime}=\left(G^{\prime}, \delta^{\prime}, \varepsilon^{\prime}\right)$ be comonads on the category $\mathbb{A}$. Then $\bar{I}: \mathbb{A}_{F} \rightarrow \mathbb{A}_{F^{\prime}}$ or $\widehat{I}: \mathbb{A}^{G} \rightarrow \mathbb{A}^{G^{\prime}}$ are liftings of the identity if the corresponding diagrams commute:
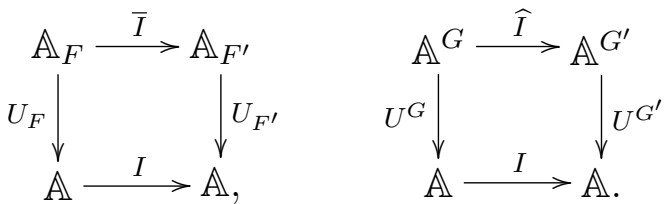

(1) There is a bijection between the liftings $\bar{I}$ of the identity functor and the monad morphisms $\alpha: F^{\prime} \rightarrow F$.

(2) There is a bijection between the liftings $\widehat{I}$ of the identity functor and the comonad morphisms $\alpha: G \rightarrow G^{\prime}$. 
Proof. The assertions follow from 3.3 and 3.5. A proof is also given in Borceux [9, Proposition 4.5.9].

The next observation is a special instance of the situation described above and is the essence of the theory of Galois comodules (see 5.9).

4.2. Adjoint functors to comodules. Let $\mathbb{G}=(G, \delta, \varepsilon)$ be a comonad on the category $\mathbb{A}$ and assume there is an adjoint pair of functors with counit

$$
L: \mathbb{B} \rightarrow \mathbb{A}^{G}, \quad R: \mathbb{A}^{G} \rightarrow \mathbb{B}, \quad \nu: L R \rightarrow I_{\mathbb{A}^{G}},
$$

for some category $\mathbb{B}$. Then the functors

$$
\widehat{R}=R \phi^{G}: \mathbb{A} \rightarrow \mathbb{B}, \quad \widehat{L}=U^{G} L: \mathbb{B} \rightarrow \mathbb{A}
$$

form an adjoint pair, we have the commutative diagram

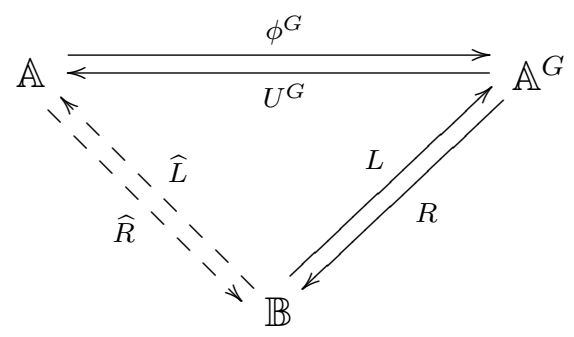

and for any $A \in \operatorname{Obj}(\mathbb{A})$ and $B \in \operatorname{Obj}(\mathbb{B})$ the isomorphisms

$$
\operatorname{Mor}_{\mathbb{A}}\left(U^{G} L(B), A\right) \simeq \operatorname{Mor}_{\mathbb{A}}^{G}\left(L(B), \phi^{G}(A)\right) \simeq \operatorname{Mor}_{\mathbb{B}}\left(B, R \phi^{G}(A)\right) .
$$

Thus the composition $\widehat{L} \widehat{R}=U^{G} L R \phi^{G}: \mathbb{A} \rightarrow \mathbb{A}$ is a comonad on $\mathbb{A}$ and there is a functorial morphism

$$
\nu_{G}(A): \widehat{L} \widehat{R}(A)=L R G(A) \rightarrow G(A),
$$

yielding the commutative diagram

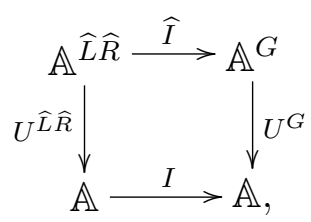

where $\widehat{I}$ is induced by the comonad morphism $\nu_{G}: \widehat{L} \widehat{R} \rightarrow G$.

In what follows we will consider the lifting of endofunctors to the category of some modules or comodules. 
4.3. Lifting of endofunctors. Let $F, G$ and $T$ be endofunctors of the category $\mathbb{A}$. For the functors $\bar{T}: \mathbb{A}_{F} \rightarrow \mathbb{A}_{F}$ and $\widehat{T}: \mathbb{A}^{G} \rightarrow \mathbb{A}^{G}$ we have the diagrams
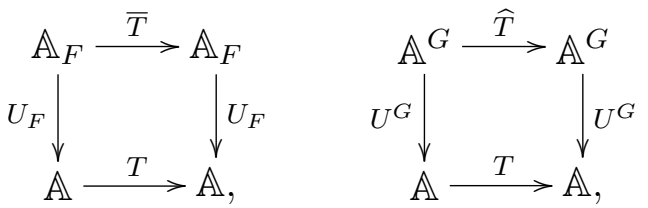

and we say that $\bar{T}$ or $\widehat{T}$ are liftings of $T$ provided the corresponding diagrams are commutative.

Besides the situations considered before we may now also ask when the liftings of a monad $T$ are again monads.

4.4. Lifting of monads to monads. Let $\mathbb{F}=(F, \mu, \eta)$ be a monad and $T: \mathbb{A} \rightarrow \mathbb{A}$ any functor on the category $\mathbb{A}$.

(1) The liftings $\bar{T}: \mathbb{A}_{\mathbb{F}} \rightarrow \mathbb{A}_{\mathbb{F}}$ of $T$ are in bijective correspondence with the natural transformations $\lambda: F T \rightarrow T F$ inducing commutativity of the diagrams
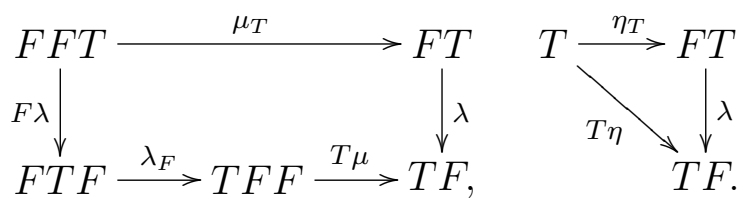

(2) If $\mathbb{T}=\left(T, \mu^{\prime}, \eta^{\prime}\right)$ is a monad, then the lifting $\bar{T}: \mathbb{A}_{\mathbb{F}} \rightarrow \mathbb{A}_{\mathbb{F}}$ of $T$ with natural transformation $\lambda: F T \rightarrow T F$ is a monad if and only if we have the commutative diagrams
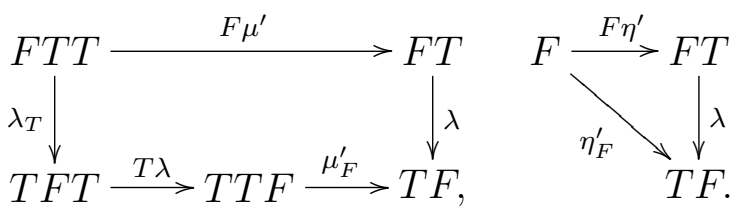

(3) For a monad $\mathbb{T}=\left(T, \mu^{\prime}, \eta^{\prime}\right)$, a natural transformation $\lambda: F T \rightarrow T F$ induces a canonical monad structure on $T F$ if and only if the diagrams in (1) and (2) are commutative.

Proof. See Beck [6]. To give an idea of the techniques involved we outline some of the arguments.

The assertion in (1) follows immediately from 3.3(1). It is related to the condition on $\lambda_{A}$ being an $F$-module morphism for any $A \in \operatorname{Obj}(\mathbb{A})$.

The diagram in (2) is derived from the requirement that $\mu_{F(A)}^{\prime}$ and $\eta_{F(A)}^{\prime}$ are to be $F$-module morphisms for any $A \in \operatorname{Obj}(\mathbb{A})$. The first of these 
conditions corresponds to commutativity of the right hand rectangle in the diagram

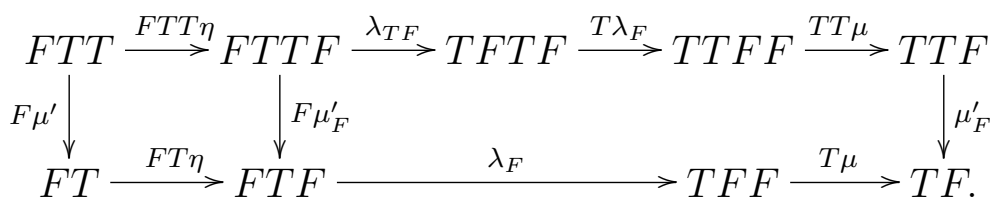

The bottom line is part of the commutative diagram

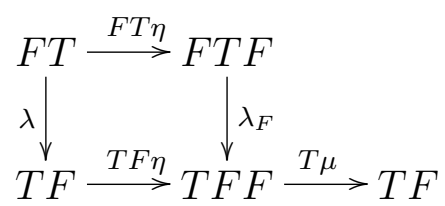

showing that $T \mu \circ \lambda_{F} \circ F T \eta=\lambda$, while the top line is part of the commutative diagram

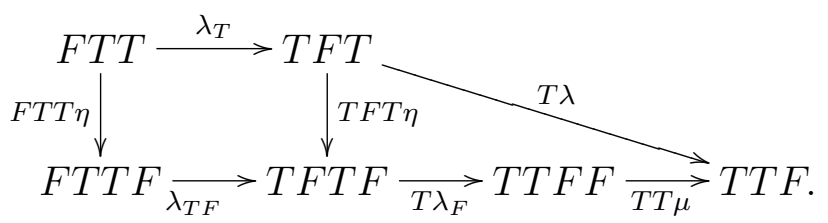

This yields the diagram given in (2).

(3) This is shown in Beck [6] who called the diagrams a distributive law of a monad $\mathbb{T}$ over a monad $\mathbb{F}$. (See also [4].)

Obviously $T F$ being a monad need not imply that $T$ and $F$ both are monads.

An explicit presentation of the material on lifting of monads and endofunctors can be found in Tanaka's thesis [50].

4.5. Definition. Given two monads $\mathbb{F}=(F, \mu, \eta)$ and $\mathbb{T}=\left(T, \mu^{\prime}, \eta^{\prime}\right)$ on a category $\mathbb{A}$, a natural transformation $\lambda: F T \rightarrow T F$ is said to be monad distributive provided the diagrams in 4.4(1) and (2) are commutative.

4.6. Tensor product of algebras. Given two $R$-algebras $A, B$, and an $R$-linear map

$$
\lambda: B \otimes_{R} A \rightarrow A \otimes_{R} B,
$$

the tensor product $A \otimes_{R} B$ can be made into an algebra by putting

$$
(a \otimes b) \cdot\left(a^{\prime} \otimes b^{\prime}\right)=a \lambda\left(b \otimes a^{\prime}\right) b^{\prime}, \quad \text { for } a, a^{\prime} \in A, b, b^{\prime} \in B .
$$

If $A$ and $B$ are associative, the functors $-\otimes_{R} A$ and $-\otimes_{R} B$ are monads on the category of $R$-modules. Then the product defined on $A \otimes_{R} B$ is associative and unital if and only if $-\otimes_{R} A \otimes_{R} B$ is a monad for the $R$-modules, that is, $\lambda$ has to induce commutativity of the corresponding diagrams in 4.4. For this special case the conditions are formulated in Caenepeel, Ion, Militaru and Zhu [18, Theorem 2.5]. 
4.7. Braidings in $R$-Mod. A prebraiding on the category $R$-Mod is given by natural morphisms

$$
\tau_{B, A}: B \otimes_{R} A \rightarrow A \otimes_{R} B
$$

for any $R$-modules $A, B$, satisfying $\tau_{A, R}=\tau_{R, A}=I_{A}$ and

$$
\tau_{B \otimes A, C}=\left(\tau_{B, C} \otimes I_{A}\right)\left(I_{B} \otimes \tau_{A, C}\right), \quad \tau_{B, A \otimes C}=\left(I_{A} \otimes \tau_{B, C}\right)\left(\tau_{B, A} \otimes I_{C}\right) .
$$

A prebraiding is called a braiding provided all $\tau_{B, A}$ are isomorphisms. If $\tau_{A, B} \circ \tau_{B, A}=I$ for all $A, B$, then the braiding $\tau$ is said to be a symmetry. Clearly the twist map tw : $B \otimes_{R} A \rightarrow A \otimes_{R} B, b \otimes a \mapsto a \otimes b$, is a symmetry.

By naturality of $\tau$, for any linear map $\mu: B \otimes_{R} B \rightarrow B$, we have the commutative diagram

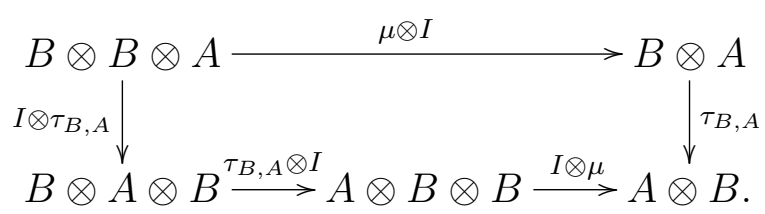

Similarly, naturality of $\tau$ induces the commutativity of all diagrams appearing in 4.4. Thus for any prebraiding $\tau$, the natural transformation

$$
-\otimes_{R} \tau_{B, A}:-\otimes_{R} B \otimes_{R} A \rightarrow-\otimes_{R} A \otimes_{R} B
$$

is monad distributive for all pairs of $R$-algebras $A, B$.

Similar constructions can be considered in monoidal categories (e.g. [28], [36], [44]).

In 4.4(2), conditions are given for the lifting of a monad to be a monad. More generally one may ask how the lifted functor $\bar{T}$ becomes a monad without $T$ being required to be a monad. Then of course some other data must be given. For an $R$-algebra $A$ and an $R$-module $V$ this was considered in Brzeziński [12, Proposition 2.1]. The transfer of this construction to endofunctors should come out as follows:

4.8. Liftings as monads. Let $\mathbb{F}=(F, \mu, \eta)$ be a monad and $T: \mathbb{A} \rightarrow \mathbb{A}$ any functor on the category $\mathbb{A}$ with a natural transformation $\iota: I \rightarrow T$ satisfying $T \iota=\iota_{T}$. Then $T F$ induces a monad $(T F, \bar{\mu}, \bar{\eta})$ on $\mathbb{A}_{\mathbb{F}}$ with unit $\bar{\eta}=T \eta \circ \iota$ and commuting diagram

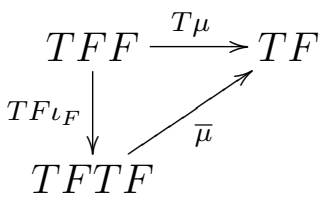


if and only if there are natural transformations

$$
\lambda: F T \rightarrow T F \text { and } \sigma: T T \rightarrow T F,
$$

such that $\lambda$ implies the commutative diagrams in 4.4(1), and for $\lambda$ and $\sigma$ there are commutative diagrams
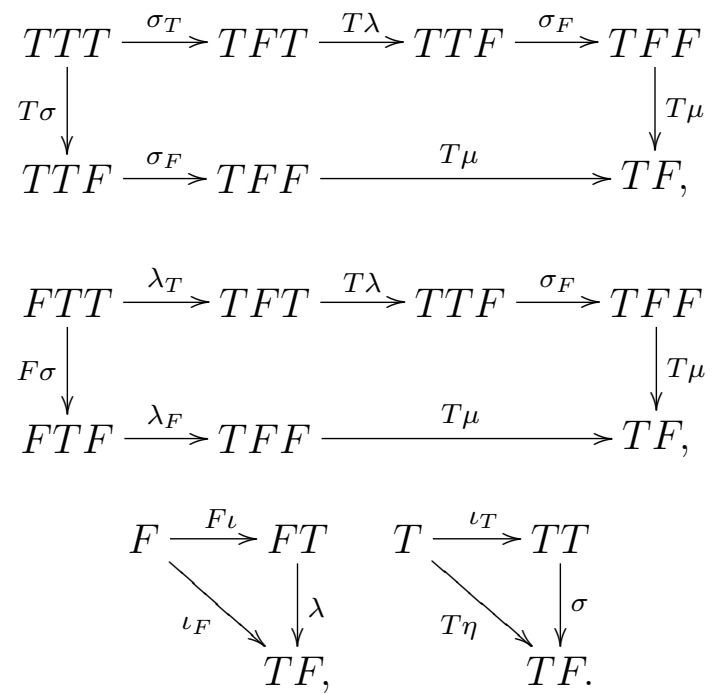

In the terminology used in [17, Definition 1.2], this means that $\sigma$ is normal (right triangle) and is a cocycle (first rectangle) satisfying the twisted module condition (second rectangle). As a special case one may take $F$ to be the identity functor. Then the conditions reduce to $T$ being a monad.

Given $\lambda$ and $\sigma$ the multiplication $\bar{\mu}$ is obtained by the diagram

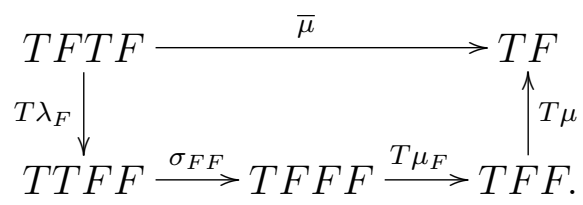

If the monad $(T F, \bar{\mu}, \bar{\eta})$ is given, suitable $\lambda$ and $\sigma$ are defined by the diagrams

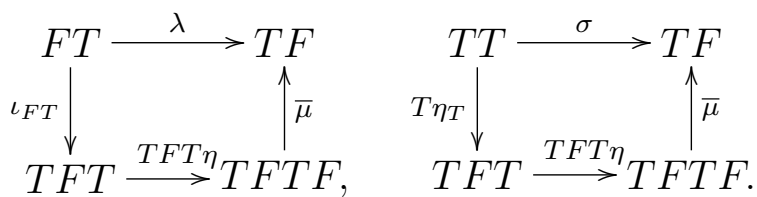

Dual to the constructions considered in 4.4 one obtains

4.9. Lifting of comonads to comonads. Let $\mathbb{G}=(G, \delta, \varepsilon)$ be a comonad and $T: \mathbb{A} \rightarrow \mathbb{A}$ any functor on the category $\mathbb{A}$. 
(1) The liftings $\widehat{T}: \mathbb{A}^{\mathbb{G}} \rightarrow \mathbb{A}^{\mathbb{G}}$ of $T$ are in bijective correspondence with the natural transformations $\varphi: T G \rightarrow G T$ inducing the commutative diagrams
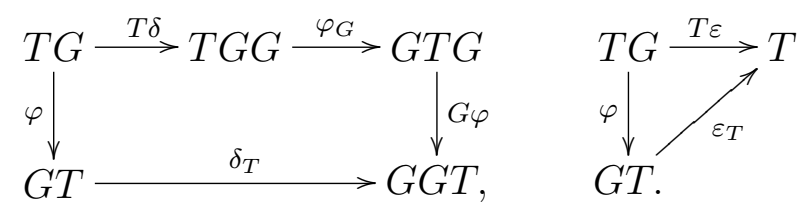

(2) If $\mathbb{T}=\left(T, \delta^{\prime}, \varepsilon^{\prime}\right)$ is a comonad, then the lifting $\widehat{T}: \mathbb{A}^{\mathbb{G}} \rightarrow \mathbb{A}^{\mathbb{G}}$ of $T$ with natural transformation $\varphi: T G \rightarrow G T$ is a comonad if and only if we have the commutative diagrams
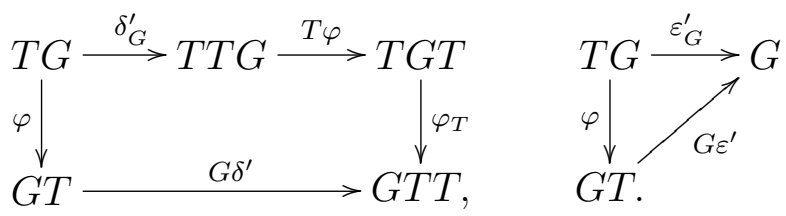

(3) For a comonad $\mathbb{T}=\left(T, \delta^{\prime}, \varepsilon^{\prime}\right)$, a natural transformation $\varphi: T G \rightarrow G T$ induces a canonical comonad structure on $T G$ if and only if the diagrams in (1) and (2) are commutative.

Proof. (1) is a special case of 3.5 and the diagram shows that $\varphi_{A}$ is a $G$-comodule morphism for any $A \in \operatorname{Obj}(\mathbb{A})$.

(2) The diagrams are derived from the conditions that $\delta_{G(A)}^{\prime}$ and $\varepsilon_{G(A)}^{\prime}$ must be $G$-comodule morphisms for all $A \in \operatorname{Obj}(\mathbb{A})$. This is seen by arguments dual to those of the proof of 4.4 .

(3) This goes back to Barr [3, Theorem 2.2].

Similar to the composition for monads, a canonical comonad structure on $T G$ need not imply that $T$ and $G$ are comonads.

4.10. Definition. Given two comonads $\mathbb{G}=(G, \delta, \varepsilon)$ and $\mathbb{T}=\left(T, \delta^{\prime}, \varepsilon^{\prime}\right)$ on a category $\mathbb{A}$, a natural transformation $\varphi: T G \rightarrow G T$ is said to be comonad distributive provided the diagrams in 4.9(1) and (2) are commutative.

4.11. Tensor product of coalgebras. Given two $R$-coalgebras $C, D$, and an $R$-linear map

$$
\varphi: C \otimes_{R} D \rightarrow D \otimes_{R} C,
$$

the tensor product $C \otimes_{R} D$ can be made into a coalgebra by putting

$$
\Delta=\left(I_{C} \otimes \varphi \otimes I_{D}\right) \circ\left(\Delta_{C} \otimes \Delta_{D}\right) .
$$

If $C$ and $D$ are coassociative, the functors $-\otimes_{R} C$ and $-\otimes_{R} D$ are comonads on the category of $R$-modules. Then the coproduct defined on $C \otimes_{R} D$ is 
coassociative and counital if and only if $-\otimes_{R} C \otimes_{R} D$ is a comonad for the $R$-modules, that is, $\varphi$ has to induce commutativity of the corresponding diagrams in 4.9. For this special case the conditions are formulated in Caenepeel, Ion, Militaru and Zhu [18, Theorem 3.4] and also in [16, 2.14].

Similar to the case of algebras (see 4.7), for a prebraiding $\tau$ on $R$-Mod and $R$-coalgebras $C, D$, the natural morphism

$$
-\otimes_{R} \tau_{C, D}:-\otimes_{R} C \otimes_{R} D \rightarrow-\otimes_{R} D \otimes_{R} C
$$

is comonad distributive (the diagrams in 4.9 commute) and thus induces a coassociative coproduct on $C \otimes_{R} D$.

In particular the twist map tw :C $C \otimes_{R} D \rightarrow D \otimes_{R} C$ satisfies the conditions imposed yielding the standard coproduct on $C \otimes_{R} D$.

4.12. Liftings as comonads. In 4.9(2), conditions are given for the lifting of a comonad to be a comonad. Dual to the case of monads one may ask how the lifted functor $\widehat{T}$ of a comonad $\mathbb{G}=(G, \delta, \varepsilon)$ becomes a comonad without $T$ being a comonad. This can be handled similar to the constructions considered in 4.8. In particular, based on a natural transformation $\varphi: T G \rightarrow G T$ satisfying 4.9(1), natural transformations $\sigma: T G \rightarrow T T$ and $\varepsilon: T \rightarrow I$ are needed satisfying appropriate conditions.

\section{Combining monads and comonads}

In this section we consider relationships between monads and comonads.

5.1. Lifting of monads for comonads. Let $\mathbb{G}=(G, \delta, \varepsilon)$ be a comonad and $T: \mathbb{A} \rightarrow \mathbb{A}$ any functor on the category $\mathbb{A}$.

(1) The liftings $\widehat{T}: \mathbb{A}^{\mathbb{G}} \rightarrow \mathbb{A}^{\mathbb{G}}$ of $T$ are in bijective correspondence with the natural transformations $\varphi: T G \rightarrow G T$ inducing the commutative diagrams
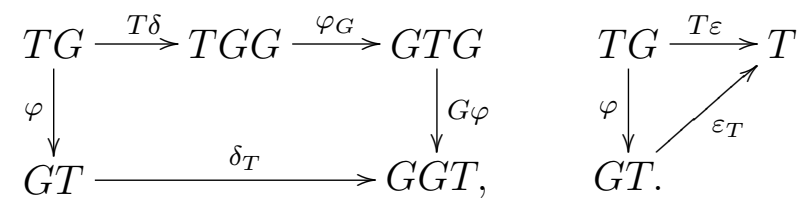

(2) If $\mathbb{T}=(T, \mu, \eta)$ is a monad, then the lifting $\widehat{T}: \mathbb{A}^{\mathbb{G}} \rightarrow \mathbb{A}^{\mathbb{G}}$ of $T$ with associated natural transformation $\varphi: T G \rightarrow G T$ is a monad if and only if we have the commutative diagrams
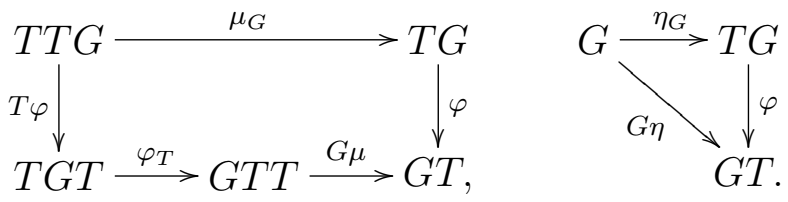
Proof. (1) follows from 3.5 and the diagrams are induced by the requirement that the $\varphi_{A}$ are $G$-comodule morphisms for all $A \in \operatorname{Obj}(\mathbb{A})$.

(2) These diagrams are consequences of the condition that $\mu_{A}$ and $\eta_{A}$ are $G$-comodule morphisms but they can also be read as condition for $\varphi_{A}$ being a $T$-module morphism for any $A \in \operatorname{Obj}(\mathbb{A})$.

5.2. Lifting of comonads for monads. Let $\mathbb{F}=(F, \mu, \eta)$ be a monad and $T: \mathbb{A} \rightarrow \mathbb{A}$ any functor on the category $\mathbb{A}$.

(1) The liftings $\bar{T}: \mathbb{A}_{\mathbb{F}} \rightarrow \mathbb{A}_{\mathbb{F}}$ of $T$ are in bijective correspondence with the natural transformations $\lambda: F T \rightarrow T F$ inducing the commutative diagrams
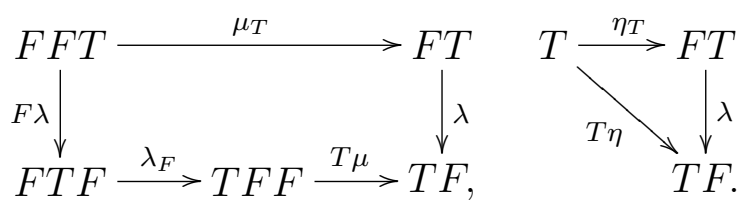

(2) If $\mathbb{T}=(T, \delta, \varepsilon)$ is a comonad, then the lifting $\bar{T}: \mathbb{A}_{\mathbb{F}} \rightarrow \mathbb{A}_{\mathbb{F}}$ of $T$ with associated natural transformation $\lambda: F T \rightarrow T F$ is a comonad if and only if we have the commutative diagrams

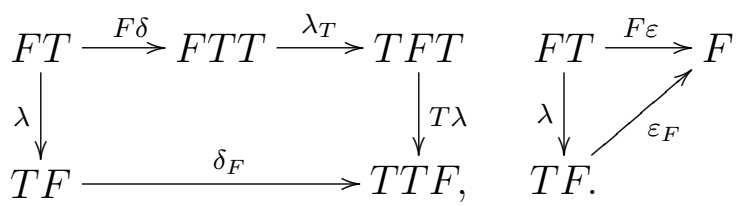

Proof. (1) follows from 3.3 and the diagrams are induced by the requirement that the $\lambda_{A}$ are $F$-module morphisms for any $A \in \operatorname{Obj}(\mathbb{A})$.

(2) These diagrams are consequences of the condition that $\delta_{A}$ and $\varepsilon_{A}$ are $F$-module morphisms but they can also be interpretted as the condition that $\lambda_{A}$ is a $T$-comodule morphism for any $A \in \operatorname{Obj}(\mathbb{A})$.

We observe that in 5.1 and 5.2 essentially the same diagrams arise.

5.3. Mixed distributive laws. Let $\mathbb{F}=(F, \mu, \eta)$ be a monad and $\mathbb{G}=$ $(G, \delta, \varepsilon)$ a comonad on the category $\mathbb{A}$. Then a natural transformation

$$
\lambda: F G \rightarrow G F
$$

is said to be mixed distributive or entwining provided it induces commutative diagrams
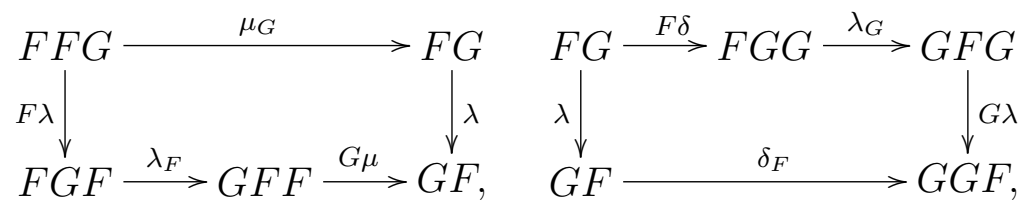


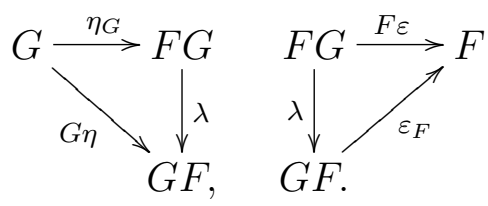

The suggestion to consider distributive laws of mixed type goes back to Beck [6, page 133] (see Remarks 5.5). The interest in these structures is based on the following theorem which follows from 5.1 and 5.2.

5.4. Characterisation of entwinings. For a monad $\mathbb{F}=(F, \mu, \eta)$ and $a$ comonad $\mathbb{G}=(G, \delta, \varepsilon)$ on the category $\mathbb{A}$, consider the diagrams
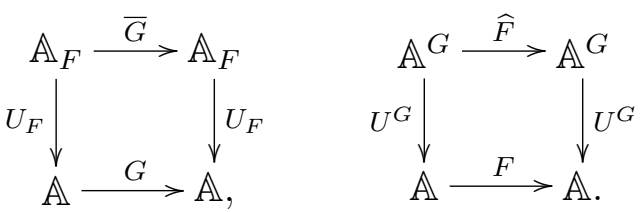

The following conditions are equivalent:

(a) There is an entwining natural transformation $\lambda: F G \rightarrow G F$;

(b) $\bar{G}: \mathbb{A}_{\mathbb{F}} \rightarrow \mathbb{A}_{\mathbb{F}}$ is a lifting of $G$ and has a comonad structure;

(c) $\widehat{F}: \mathbb{A}^{\mathbb{G}} \rightarrow \mathbb{A}^{\mathbb{G}}$ is a lifting of $F$ and has a monad structure.

5.5. Remarks. The preceding theorem was first formulated 1973 by van Osdol in [54, Theorem IV.1]. It was extended to $\mathcal{V}$-categories in Wolff [57, Theorem 2.4] and was rediscovered in 1997 by Turi and Plotkin in the context of operational semantics in [52, Theorem 7.1]. In the same year the corresponding notion for tensor functors was considered by Brzeziński and Majid who coined the name entwining structure for a mixed distributive law for an algebra $A$ and a coalgebra $C$ over a commutative ring $R$ in [14, Definition 2.1] (see 5.8). The connection between this notions is also mentioned in Hobst and Pareigis [26].

It was observed by Takeuchi that these structures are closely related to corings (see [13, Proposition 2], [16, 32.6]). This is a special case of 5.4(b) since the coring $A \otimes_{R} C$ is just a comonad on the category of right $A$-modules. The comultiplication is a special case of the constructions considered in the next section. Similarly, by $5.4(\mathrm{c}), C \otimes_{R} A$ can be seen as a monad on the category of right $C$-comodules.

5.6. Comultiplication induced by units. Let $F, G$ be endofunctors on a category $\mathbb{A}$ and $\eta: I \rightarrow F$ a natural transformation. Then we have natural transformations

$$
\eta_{G}: G \rightarrow F G, \quad G \eta: G \rightarrow G F
$$


and naturality of $\eta$ implies commutativity of the diagrams

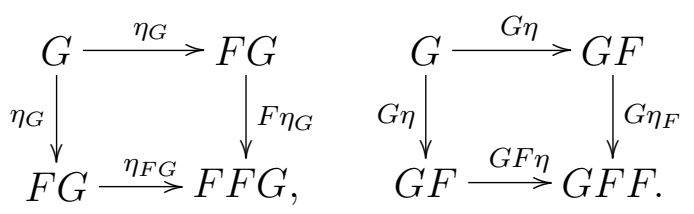

For $F=G$ the diagrams show that both $\eta_{F}$ and $F \eta$ induce coassociative comultiplications on $F$.

If there is a coassociative comultiplication $\delta: G \rightarrow G G$, then we can define a comultiplication on $F G$ by

$$
\bar{\delta}: F G \stackrel{F \delta}{\longrightarrow} F G G \stackrel{F G \eta_{G}}{\longrightarrow} F G F G,
$$

which is coassociative by commutativity of the diagram

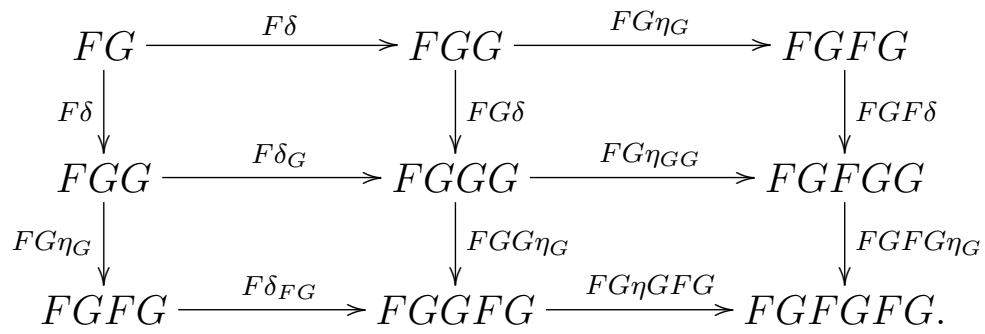

The left top rectangle commutes by coassociativity of $\delta$, the right top rectangle by naturality of $\eta$, the left bottom rectangle by naturality of $\delta$ and the right bottom rectangle again by naturality of $\eta$.

For a monad $\mathbb{F}=(F, \mu, \eta)$ the comultiplication on $F G$ can also be derived from general properties of adjoint functors.

Symmetrically a coassociative comultiplication for $G F$ is defined by

$$
\tilde{\delta}: G F \stackrel{\delta_{F}}{\longrightarrow} G G F \stackrel{G \eta_{G F}}{\longrightarrow} G F G F .
$$

In case a natural transformation $\varepsilon: G \rightarrow I$ is given, we have natural transformations $\varepsilon_{F}: G F \rightarrow F$ and $F \varepsilon: F G \rightarrow F$ allowing to dualise the above constructions. Then an associative multiplication $\mu: F F \rightarrow F$ induces associative multiplications on $G F$ and $F G$.

Now let $\mathbb{F}=(F, \mu, \eta)$ be a monad and $\mathbb{G}=(G, \delta, \varepsilon)$ a comonad on $\mathbb{A}$ with a natural transformation $\lambda: F G \rightarrow G F$ satisfying $\lambda \circ \eta_{G}=G \eta$ (left triangle in 5.3). Then we have the commutative diagram

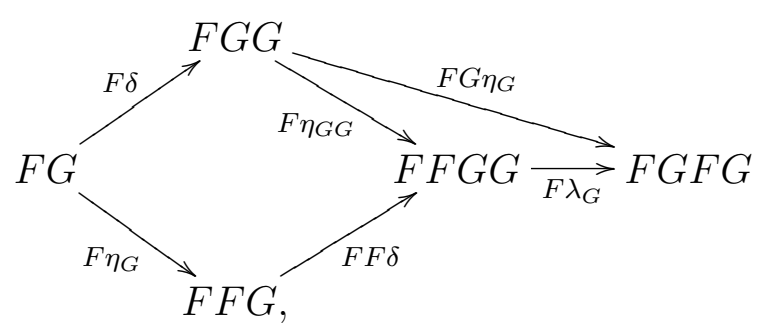


showing that the coproduct on $F G$ induced by an entwining $\lambda$ is the same as the one considered above.

5.7. Mixed bimodules. Given a monad $\mathbb{F}=(F, \mu, \eta)$ and a comonad $\mathbb{G}=(G, \delta, \varepsilon)$ on the category $\mathbb{A}$ with an entwining $\lambda: F G \rightarrow G F, \lambda$-bimodules or mixed bimodules are defined as those $A \in \operatorname{Obj}(\mathbb{A})$ with morphisms

$$
F(A) \stackrel{h}{\longrightarrow} A \stackrel{k}{\longrightarrow} G(A)
$$

such that $(A, h)$ is an $\mathbb{F}$-module and $(A, k)$ is a $\mathbb{G}$-comodule satisfying the pentagonal law

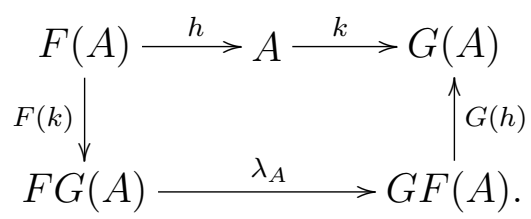

A morphism $f: A \rightarrow A^{\prime}$ between two $\lambda$-bimodules is a bimodule morphism provided it is both an $F$-module and a $G$-comodule morphism.

These notions yield the category of $\lambda$-bimodules which we denote by $\mathbb{A}_{F}^{G}$. This category can also be considered as the category of $\widehat{G}$-comodules for the comonad $\widehat{G}: \mathbb{A}_{F} \rightarrow \mathbb{A}_{F}$ and also as the category of $\bar{F}$-modules for the monad $\bar{F}: \mathbb{A}^{G} \rightarrow \mathbb{A}^{G}$ (e.g. $[52,7.1]$ ). For every $F$-module $A, G(A)$ is a $\lambda$-bimodule and for any $G$-comodule $A^{\prime}, F\left(A^{\prime}\right)$ is a $\lambda$-bimodule canonically. In particular, for every $A \in \operatorname{Obj}(\mathbb{A}), F G(A)$ and $G F(A)$ are $\lambda$-bimodules.

As a sample we draw the diagram showing that, for any $F$-module $\varrho_{A}$ : $F(A) \rightarrow A, G(A)$ is a $\lambda$-bimodule with module structure given by the composition $G \rho_{A} \circ \lambda_{A}: F G(A) \rightarrow G(A)$ :

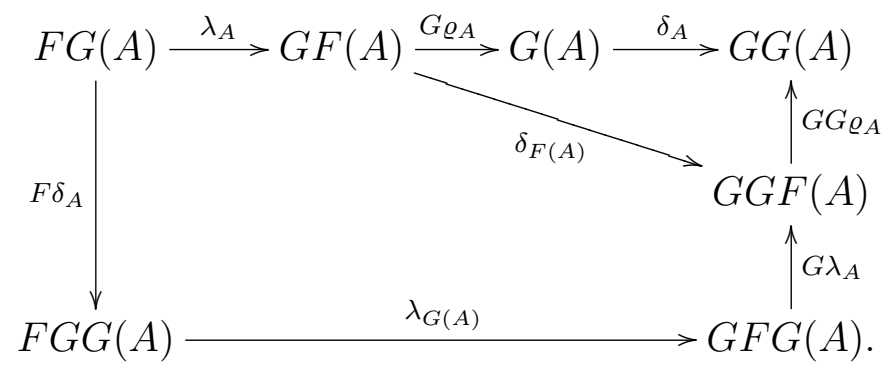

The triangle is commutative by naturality of $\delta$, the pentagon is commutative by one of the mixed distributive laws.

5.8. Entwined algebras and coalgebras. Given an $R$-algebra $(A, \mu, \eta)$ and an $R$-coalgebra $(C, \Delta, \varepsilon)$, the functor $-\otimes_{R} A$ is a monad and $-\otimes_{R} C$ is a comonad on the category of $R$-modules. 
If the functor $-\otimes_{R} C$ can be lifted to the $A$-modules (equivalently $-\otimes_{R} A$ can be lifted to the $C$-comodules) then there is an $R$-linear map

$$
\psi: C \otimes_{R} A \rightarrow A \otimes_{R} C,
$$

and the diagrams in 5.3 yield the conditions for an entwining structure introduced by Brzeziński and Majid in [14] (see bow-tie diagram in [16, 32.2]):

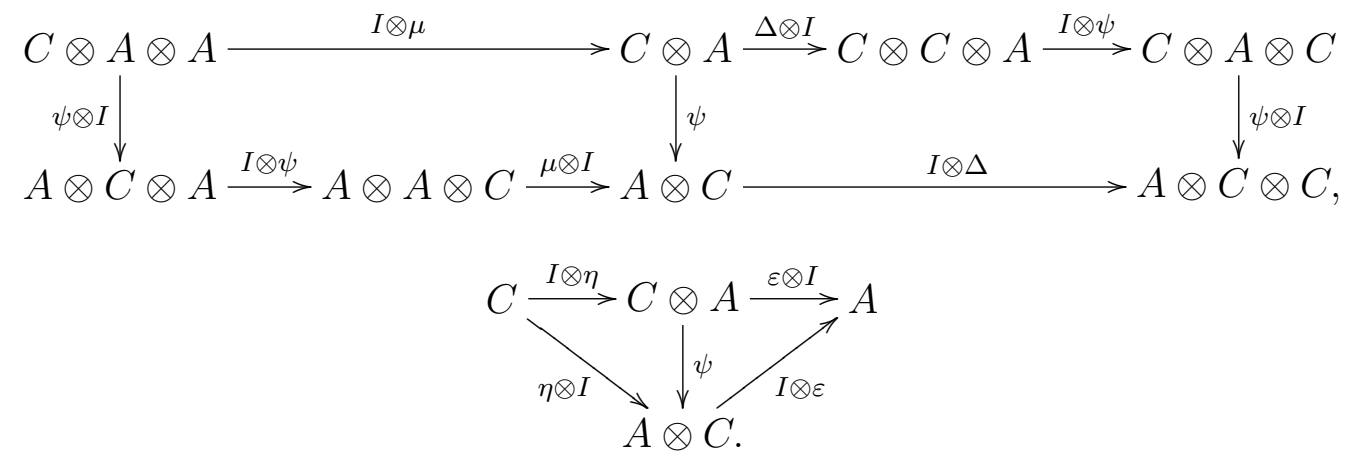

A comultiplication on $A \otimes_{R} C$ is defined by the general formalism considered in 5.6 making $A \otimes_{R} C$ an $A$-coring.

Let $M$ be an $R$-module with an $A$-module structure $\varrho_{M}: M \otimes_{R} A \rightarrow M$ and a $C$-comodule structure $\varrho^{M}: M \rightarrow M \otimes_{R} C$. Then $M$ is an entwined module if the diagram

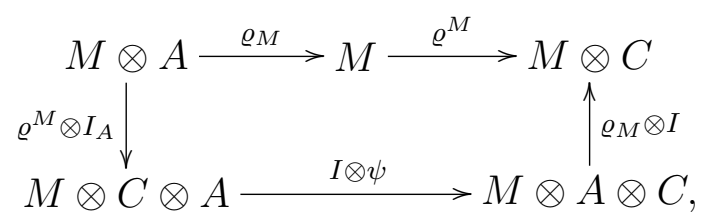

is commutative (e.g. $[16,32.4])$. This means that $\varrho_{M}$ is a comodule morphism when $M \otimes_{R} A$ is considered as a $C$-comodule with structure map $\left(I_{M} \otimes \psi\right) \circ$ $\left(\varrho^{M} \otimes I_{A}\right)$, and $\varrho^{M}$ is an $A$-module morphism when $M \otimes_{R} C$ is an $A$-module with structure map $\left(\varrho_{M} \otimes I_{C}\right) \circ\left(I_{M} \otimes \psi\right)$. Observe the interplay between these structures: given an entwining $\psi$ the diagram imposes conditions on $\varrho_{M}$ or $\varrho^{M}$. On the other hand, if these two morphisms are given the problem is to find a suitable $\psi$.

Notice that $A$ need not be a $C$-comodule unless it has a grouplike element. For more details the reader may consult [16, Section 32].

A braiding on the category of entwined modules induced by a morphism $C \otimes_{R} C \rightarrow A \otimes_{R} A$ is considered by Hobst and Pareigis in [26, Theorem 5.5].

5.9. Galois comodules. Let $\mathcal{C}$ be a coring over a ring $A$ and $P \in \mathbf{M}^{\mathcal{C}}$ with $S:=\operatorname{End}^{G} P$. Then there is an adjoint pair of functors

$$
-\otimes_{S} P: \mathbf{M}_{S} \rightarrow \mathbf{M}^{\mathcal{C}}, \quad \operatorname{Hom}^{\mathcal{C}}(P,-): \mathbf{M}^{\mathcal{C}} \rightarrow \mathbf{M}_{S}
$$


with counit ev $: \operatorname{Hom}^{\mathcal{C}}(P,-) \otimes_{S} P \rightarrow I_{\mathbf{M}^{\mathcal{c}}}$, and, by 4.2 , there is a functorial morphism

$$
\mathrm{ev}_{\mathcal{C}}: \operatorname{Hom}_{A}(P,-) \otimes_{S} P \rightarrow-\otimes_{A} \mathcal{C} .
$$

$P$ is called a Galois comodule provided $\mathrm{ev}_{\mathcal{C}}$ is an isomorphism. For further details about these comodules we refer to [56].

5.10. Bialgebras and Hopf modules. Consider an $R$-module $B$ which is both an $R$-algebra $\mu: B \otimes_{R} B \rightarrow B, \eta: R \rightarrow B$, and an $R$-coalgebra $\Delta: B \rightarrow B \otimes_{R} B, \varepsilon: B \rightarrow R$. Define a linear map $\psi$ by commutativity of the diagram

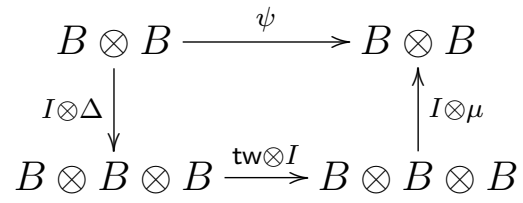

which produces

$$
\psi: B \otimes_{R} B \rightarrow B \otimes_{R} B, \quad a \otimes b \mapsto(1 \otimes a) \Delta(b) .
$$

To make $B$ a bialgebra, $\mu$ and $\eta$ must be coalgebra maps (equivalently, $\Delta$ and $\varepsilon$ are to be algebra maps) with respect to the obvious product and coproduct on $B \otimes_{R} B$ (induced by tw). This can be expressed by commutativity of the set of diagrams
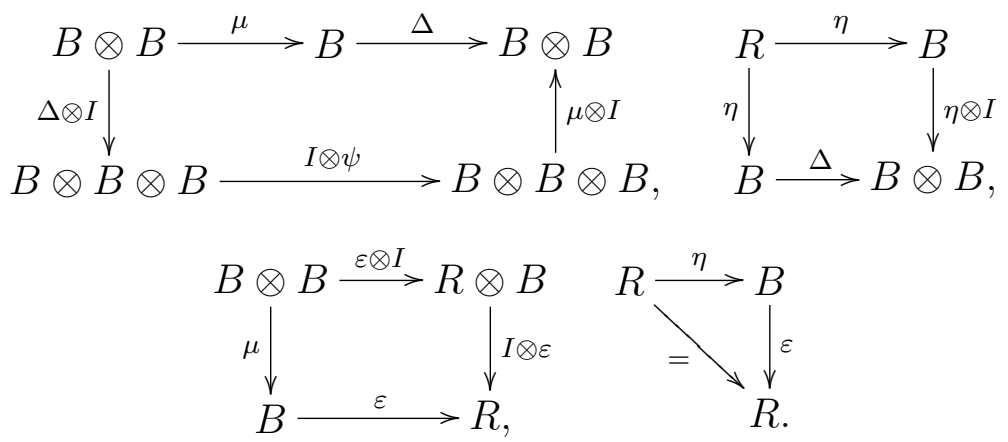

These show that $\varepsilon$ is a monad morphism and $\eta$ is a comonad morphism, and $\mu$ is a right $B$-comodule morphism when $B \otimes_{R} B$ is considered as right $B$-comodule by $(I \otimes \psi) \circ(\Delta \otimes I)$. They also imply that every $R$-module $M$ is a $B$-module and $B$-comodule trivially by $I \otimes \varepsilon: M \otimes_{R} B \rightarrow M$ and $I \otimes \eta: M \rightarrow M \otimes_{R} B$.

If the above conditions hold then it is easily checked that the given $\psi$ is an entwining and $B$ is called a $(\psi$-)bialgebra. Similarly, for any entwining $\psi^{\prime}: B \otimes_{R} B \rightarrow B \otimes_{R} B$ one may define $\psi^{\prime}$-bialgebras. Certainly, the twist tw is an entwining but $B$ is only a tw-bialgebra provided $\Delta$ is trivial, that is, $\Delta(b)=b \otimes 1$ for any $b \in B$. 
An $R$-module $M$ which is both a $B$-module $\varrho_{M}: M \otimes_{R} B \rightarrow M$ and a $B$-comodule $\varrho^{M}: M \rightarrow M \otimes_{R} B$ is called a $(\psi$ - $) B$-bimodule or a $B$-Hopf module if the diagram

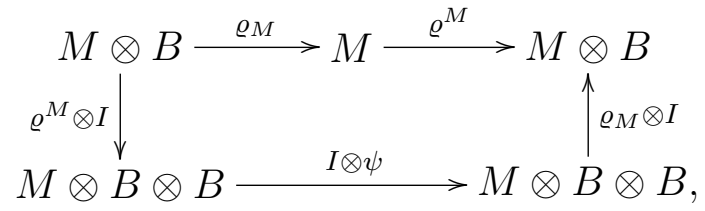

is commutative. In this case $B$ is a right $B$-bimodule and we have the commutative diagram

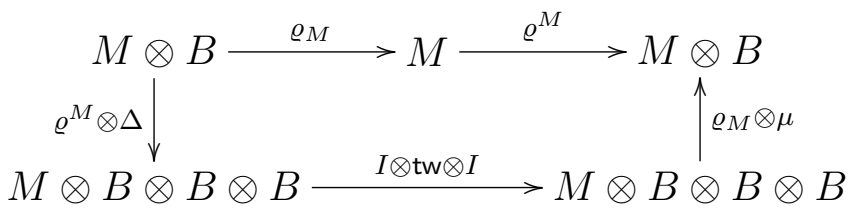

which holds in particular for $M=B$.

Here we have derived our constructions from the twist tw but the same pattern can be followed starting with a (pre-)braiding on $R$-Mod (or on a monoidal category, e.g. [44]).

5.11. Hopf algebras. The bialgebra $B$ is a Hopf algebra if (one of) the following equivalent conditions hold (e.g. [16, 15.5]):

(a) $B$ has an antipode $S: B \rightarrow B$;

(b) the functor $B \otimes_{R}-: \mathbf{M}_{R} \rightarrow \mathbf{M}_{B}^{B}$ is an equivalence;

(c) the functor $\operatorname{Hom}_{B}^{B}(B,-): \mathbf{M}_{B}^{B} \rightarrow \mathbf{M}_{R}$ is an equivalence;

(d) $B$ is a Galois comodule for the coring $B \otimes_{R} B$ (in the sense of 5.9).

If $B$ is flat as an $R$-module, then (a)-(d) are equivalent to:

(e) $B$ is a (projective) generator in $\mathbf{M}_{B}^{B}$.

Being on the subject we mention the algebra counterpart of Hopf algebras:

5.12. Azumaya algebras. A central $R$-algebra $A$ is an Azumaya algebra if (one of) the following equivalent conditions hold:

(a) $A \otimes_{R}{ }_{A} \operatorname{Hom}_{A}\left(A, A \otimes_{R} A\right) \rightarrow A \otimes_{R} A,(a, f) \mapsto f(a)$, is an isomorphism;

(b) the functor $A \otimes_{R}-: \mathbf{M}_{R} \rightarrow{ }_{A} \mathbf{M}_{A}$ is an equivalence;

(c) the functor ${ }_{A} \operatorname{Hom}_{A}(A,-):{ }_{A} \mathbf{M}_{A} \rightarrow \mathbf{M}_{R}$ is an equivalence;

(d) $A$ is a (projective) generator in ${ }_{A} \mathbf{M}_{A}$.

An extension (in a different vein) of this notion to Azumaya categories is proposed by Borceux and Vitale in [10]. 
The formalism outlined for Hopf algebras can be transferred to endofunctors on arbitrary categories $\mathbb{A}$.

5.13. Mixed bimonads. An endofunctor $B: \mathbb{A} \rightarrow \mathbb{A}$ is called a mixed bimonad or just bimonad if it has a monad structure $\mathbb{B}=(B, \mu, \eta)$ and a comonad structure $\mathbb{B}=(B, \delta, \varepsilon)$ with an entwining functorial morphism

$$
\psi: B B \rightarrow B B
$$

and commutative diagrams
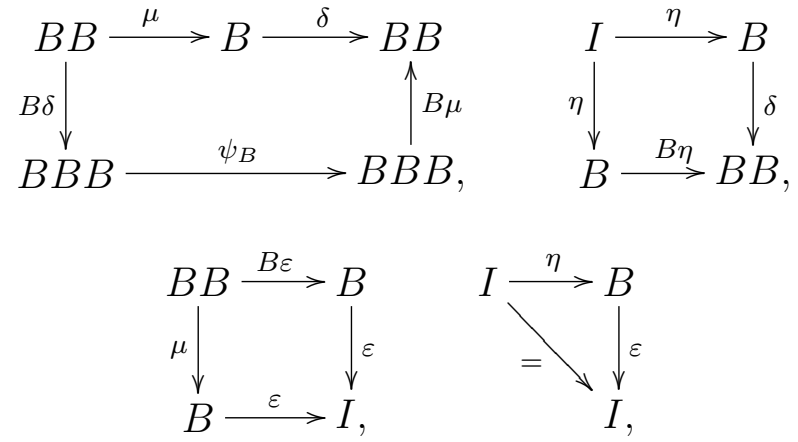

making $\eta$ a comonad morphism and $\varepsilon$ a monad morphism.

The (mixed) $B$-bimodules $A$ are $B$-modules and $B$-comodules satisfying the pentagonal law

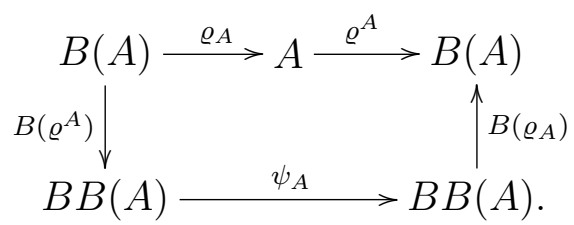

$B$-bimodule morphisms are both $B$-module and $B$-comodule morphisms. We denote the induced category by $\mathbb{A}_{B}^{B}$.

Any object $A$ in $\mathbb{A}$ is a $B$-module and $B$-comodule trivially by the morphisms $\varepsilon_{A}: B(A) \rightarrow A$ and $\eta_{A}: A \rightarrow B(A)$ but need not be a $B$-bimodule.

The commutativity of the first diagram in the definition ensures that for every $A \in \operatorname{Obj}(\mathbb{A}), B(A)$ is a $B$-bimodule canonically and hence we have a functor

$$
\begin{aligned}
& \phi_{B}^{B}: \mathbb{A} \rightarrow \mathbb{A}_{B}^{B}, \quad A \mapsto B B(A) \stackrel{\mu_{A}}{\rightarrow} B(A) \stackrel{\delta_{A}}{\rightarrow} B B(A), \\
& A \stackrel{f}{\rightarrow} A^{\prime} \mapsto B(A) \stackrel{B(f)}{\rightarrow} B\left(A^{\prime}\right) .
\end{aligned}
$$

By the monad and comonad relations involved we get, for any $A, A^{\prime} \in \operatorname{Obj}(\mathbb{A})$, the functorial isomorphisms

$$
\operatorname{Mor}_{B}^{B}\left(B(A), B\left(A^{\prime}\right)\right) \simeq \operatorname{Mor}_{B}\left(B(A), A^{\prime}\right) \simeq \operatorname{Mor}_{\mathbb{A}}\left(A, A^{\prime}\right)
$$


which show that the functor $\phi_{B}^{B}$ is full and faithful. This was observed for Hopf modules in braided monoidal categories by Bespalov and Drabant in [7, Proposition 3.3.2].

5.14. Bimonads induced by monads. Let $\mathbb{B}=(B, \mu, \eta)$ be a monad on a category $\mathbb{A}$ and consider the coproduct $B \eta: B \rightarrow B B$ (see 5.6). Let $\lambda: B B \rightarrow B B$ be any natural transformation which is monad distributive for $B$ (see 4.5). Then, by $4.4(2), \lambda \circ B \eta=\eta_{B}$, and this yields commutativity of the diagram

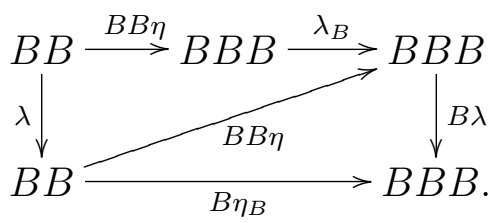

Thus the two rectangles needed for a mixed distributive law are commutative. Here we have no counit for $(B, B \eta)$.

The compatibility condition holds for the product $\mu: B B \rightarrow B$ and the coproduct $\lambda \circ B \eta: B \rightarrow B B$ by commutativity of the diagram

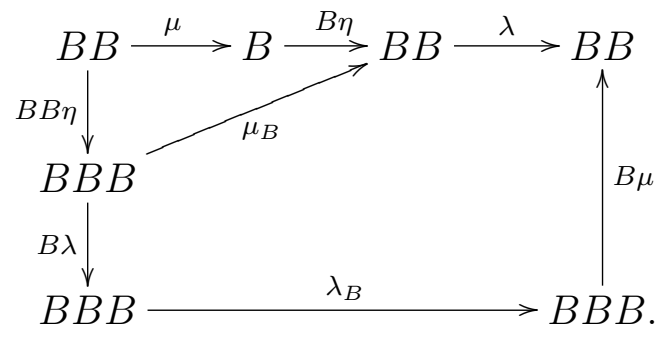

The triangle is commutative by naturality of $\mu$ and the pentagon is just the diagram from 4.4(1).

A map $\psi$ will be defined in $5.17(2)$, by commutativity of the outer rectangle in the diagram

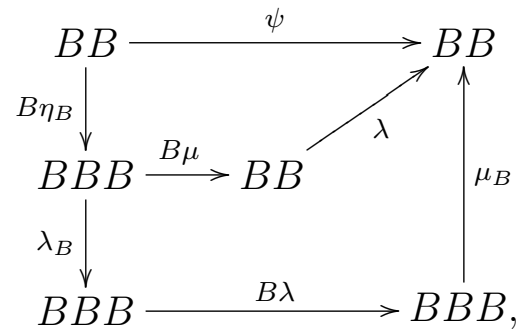

Since the pentagon is commutative by $4.4(2)$ and $\mu \circ \eta_{B}=I_{B}$ we obtain that $\psi=\lambda$ in this case.

5.15. Hopf monads. From the characterisations of Hopf algebras in 5.11, condition (b) can be formulated in full generality. Thus one may call the 
bimonad $B$ a Hopf monad if the functor $\phi_{B}^{B}$ (from 5.13) induces an equivalence of categories.

Obviously the classical Hopf algebras over commutative rings are examples for this situation and the question arises if a Hopf monad can be characterised by some other of the characterisations cited in 5.11. It should be no problem to define a (left and right) antipode as a natural morphism $S: B \rightarrow B$, with the properties

$$
\mu \circ S_{B} \circ \delta=\eta \circ \varepsilon=\mu \circ B S \circ \delta .
$$

However, it is not clear under which premisses the existence of such an antipode implies the fundamental theorem, that is, that $\phi_{B}^{B}$ is an equivalence. In monoidal categories in which the tensor product preserves coequalisers, this is outlined by Mesablishvili in [40]. To prove this for module categories, the twist map plays a central role. In monoidal categories this can be replaced by a more general braiding and it is shown in Bespalov and Drabant in [7] that in braided monoidal categories the existence of an antipode implies the fundamental theorem provided idempotents split in the base category. There is some hope to get a similar result in not necessarily braided categories in view of the following observations.

5.16. Braided bialgebras. The notions developed in 5.10 were derived from the twist or a braiding in the category of $R$-modules and one may ask if similar conditions can be considered in the general case. Initially braided Hopf algebras were placed in braided categories ([36], [44]). Takeuchi suggested in [49, Definition 5.1] to define braided bialgebras $H$ by introducing a YangBaxter operator $R: H \otimes_{R} H \rightarrow H \otimes_{R} H$ and requiring some compatibility conditions including that $R$ is monad and comonad distributive. Thus, in contrast to other generalisations of braided Hopf algebras, he did not refer to the braiding on the base category (of vector spaces). Similar situations were studied by Menini and Stefan in [38] (see 5.18), the Gucciones in [22], and Kharchenko in [30]. Another view on the relations between Yang-Baxter operators and entwining structures is given by Brzeziński and Nichita in [15].

The conditions for a braided bialgebra given in [49, Definition 5.1] may be adapted in the following way.

5.17. Braided bimonad. Let $\mathbb{B}=(B, \mu, \eta, \delta, \varepsilon)$ be a monad and comonad on a category $\mathbb{A}$. Consider a natural (iso-)morphism $\tau: B B \rightarrow B B$ satisfying the Yang-Baxter (or braid) equation

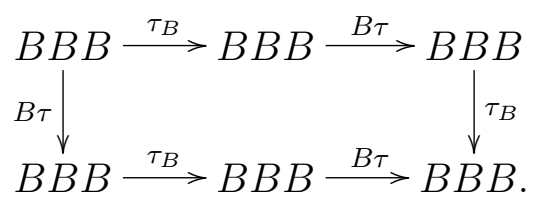

$\mathbb{B}$ is said to be a braided bimonad if 
(1) $\tau$ is monad and comonad distributive for $B$ (see 4.5, 4.10);

(2) $\mathbb{B}$ is a mixed bimonad for $\psi$ defined (as in 5.10) by

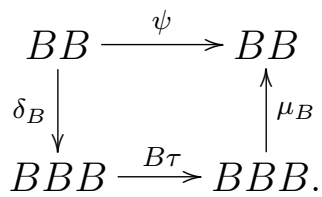

The resulting formalism is similar to that considered in 5.10. In particular we obtain the commutative diagram

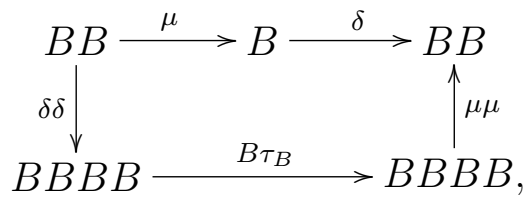

a condition for bialgebras in prebraided categories (e.g. Schauenburg [44]).

5.18. Remarks. Conditions of similar type are required by Menini and Stefan in [38, Definition 1.3] to define a compatible flip morphism. Variations of their conditions are considered by Kasangian, Lack and Vitale in [29], where it is shown that compatible flip morphisms are (monad distributive and) involutive $B C D$-laws in their terminology. In these papers only monads are considered and hence no monad-comonad compatibility comes in. However, as shown in 5.14 , any monad $(B, \mu, \eta)$ with a monad distributive natural transformation $\tau: B B \rightarrow B B$ has a compatible comonad structure (without counit) and thus many of the conditions required in 5.17 are satisfied for the coproduct $\tau \circ B \eta$ (compare also [29, Section 3]). In this case the morphism $\psi$ is equal to $\tau$ (see last diagram in 5.14).

The conditions required in 5.17 may be also weakened, for example, by deleting some of the restraints for the unit or counit. This is done, for example, by van Daele and Wang in [53] in braided monoidal categories by defining unifying braided bialgebras (and Hopf algebras). For further work in this direction the reader may consult [55], Böhm, Nill, and Szlachány [8], and Caenepeel, Wang, and Yin [20].

Given the data for a braided bimonad it may be possible to adapt the arguments from [7] to derive for (braided) bimonads the fundamental theorem from the existence of an antipode $S: B \rightarrow B$ (see 5.13) for (not necessarily monoidal) categories with splitting idempotents. Then the coinvariants of a mixed $B$-bimodule $X$ should be defined by the splitting of $X \rightarrow B(X) \stackrel{S_{X}}{\longrightarrow}$ $B(X) \rightarrow X$ (see [7, Proposition 3.2.1]).

Similar questions are handled in connection with Turaev's Hopf groupcoalgebras by Caenepeel and de Lombaerde in [19]. They consider the example of a Hopf algebra in the category of sets (not a module category but 
still monoidal and braided by product and twist). To illustrate our formalism and as a pattern for more general examples we sketch this in the terminology used here and extend the list of characterisations of groups.

5.19. Hopf monads on the category of sets. For a set $G$, consider the endofunctor in the category Set, $\bar{G}=G \times-$ with $\bar{G}(X)=G \times X$ for any set $X . \bar{G}$ is a comonad if $G$ is a coalgebra by the (unique) structure maps

$$
\delta: G \rightarrow G \times G, g \mapsto(g, g) \text { and } \varepsilon: G \rightarrow I, g \mapsto\{\omega\}
$$

where $\{\omega\}$ is a singleton (see [19, Lemma 1.3], [48, 4.6]).

Following our terminology the category of $\bar{G}$-comodules is denoted by $\operatorname{Set}^{G}$. For $A, B \in \operatorname{Set}^{G}$, the $\bar{G}$-morphisms are written as $\operatorname{Map}^{G}(A, B)$ and there is a bijection

$$
\operatorname{Map}^{G}(A, G \times B) \rightarrow \operatorname{Map}(A, B), \quad f \mapsto(\varepsilon \times I) \circ f .
$$

It follows by counitality that, for any $G$-comodule $\varrho^{G}: X \rightarrow G \times X$, we can write

$$
\varrho^{G}(x)=(\rho(x), x), \text { for some } \rho \in \operatorname{Map}(X, G) .
$$

$\bar{G}$ is a monad provided $(G, \mu, \eta)$ is a semigroup. The objects of the category $\operatorname{Set}_{G}$ of $\bar{G}$-modules are known as $G$-sets. As usual, for a $G$-module $\varrho_{G}: G \times X \rightarrow X$ we write $\varrho_{G}(g, x)=g x$, for $g \in G$ and $x \in X$.

$\operatorname{Map}_{G}(A, B)$ denotes the $G$-morphisms between sets $A, B$ in $\operatorname{Set}_{G}$ and there is a bijection

$$
\operatorname{Map}_{G}(G \times A, B) \rightarrow \operatorname{Map}(A, B), \quad f \mapsto f \circ \eta_{A},
$$

in particular the bijection

$$
\varphi_{A}: \operatorname{Map}_{G}(G, A) \rightarrow A, \quad \varphi_{A}(f)=f\left(1_{G}\right), \quad \varphi_{A}^{-1}(a)=[g \mapsto g a] .
$$

Referring to the twist map we define an entwining $G G \rightarrow G G$ as in 5.17,

$$
\psi=(\mu \times I) \circ(I \times \mathrm{tw}) \circ(\delta \times I): G \times G \rightarrow G \times G, \quad(g, h) \mapsto(g h, g) .
$$

For this, the monad and comonad structures on $G$ are compatible (diagrams in 5.13) and thus we have a mixed bimonad in the sense of 5.13.

Mixed $\bar{G}$-bimodules are defined as sets $X$ with $G$-action $\varrho_{X}: G \times X \rightarrow X$ and $G$-coaction $\varrho^{X}: X \rightarrow G \times X$ and the compatibility condition from 5.13 requires for $g \in G$ and $x \in X$ the equality

$$
\varrho^{X}(g x)=(\rho(g x), g x)=(g \rho(x), g x) .
$$

Thus, as pointed out in [19], mixed $\bar{G}$-bimodules are $G$-sets $X$ with some map $\rho: X \rightarrow G$ satisfying 


$$
\rho(g x)=g \rho(x) \text { for all } g \in G \text { and } x \in X .
$$

For any sets $X$ and $Y$ there are bijections

$$
\operatorname{Map}_{G}^{G}(G \times X, G \times Y) \rightarrow \operatorname{Map}_{G}(G \times X, Y) \rightarrow \operatorname{Map}(X, Y) .
$$

For any mixed $\bar{G}$-bimodule $A$ there is the bijection,

$$
\varphi_{A}: \operatorname{Map}_{G}^{G}(G, A) \rightarrow A^{c o G}=\left\{a \in A \mid \varrho^{A}(a)=\left(1_{G}, a\right)\right\}=\left\{a \in A \mid \rho(a)=1_{G}\right\},
$$

where the $G$-coinvariants come out as in [19], with inverse map

$$
\varphi_{A}^{-1}: A^{c o G} \rightarrow \operatorname{Map}_{G}^{G}(G, A), \quad c \mapsto[g \mapsto g c] .
$$

Considering $G \times G$ as $\bar{G}$-bimodule with coaction $\varrho^{G G}(x, y)=(x, x, y)$ and action $\varrho_{G G}(g,(x, y))=(g x, g y)$, for $g, x, y \in G$, we obtain the bijection

$$
\varphi_{G G}: \operatorname{Map}_{G}^{G}(G, G \times G) \rightarrow\left\{1_{B}\right\} \times G \simeq G
$$

with inverse map

$$
\varphi_{G G}^{-1}: G \simeq\left\{1_{G}\right\} \times G \rightarrow \operatorname{Map}_{G}^{G}(G, G \times G), \quad h \mapsto\left(1_{G}, h\right) \mapsto[g \mapsto(g, g h)] .
$$

The evaluation map now yields the commutative diagram

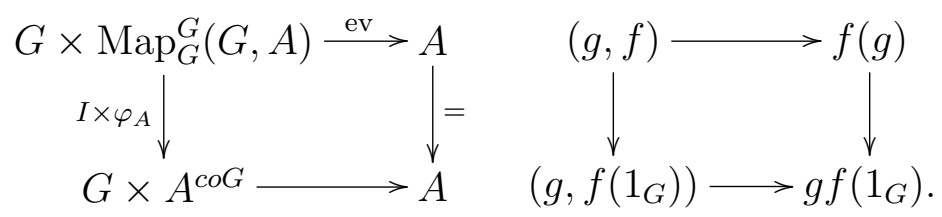

In particular, for $A=G \times G$ the canonical map is defined by the diagram

$$
\begin{aligned}
& \operatorname{Map}_{G}^{G}(G, G \times G) \times G \stackrel{\mathrm{ev}}{\longrightarrow} G \times G \\
& \varphi_{G G} \times I \downarrow \\
& G \times G \longrightarrow \gamma_{G} \longrightarrow G \times G, \\
& \gamma_{G}=\mathrm{ev} \circ\left(\varphi_{G G}^{-1} \times I\right): G \times G \rightarrow G \times G, \quad(g, h) \mapsto(g, g h) .
\end{aligned}
$$

An antipode $S: \bar{G} \rightarrow \bar{G}$ should come from a map $s: G \rightarrow G$ and the conditions (as stated in 5.15) imply

$$
g s(g)=1_{G}=s(g) g \text { for all } g \in G,
$$

and thus an antipode exists if and only if the semigroup $G$ is a group ([19, Lemma 1.3]). Notice that in this case already one of the equalities (for all $g \in G)$ is enough to imply that $G$ is a group. 
If the canonical map is bijective we can follow the arguments in $[16,15.2]$ to show that an antipode exists.

So assume the canonical map $\gamma: G \times G \rightarrow G \times G$ as defined above to have a left inverse $\beta: G \times G \rightarrow G \times G$ respecting the right $G$-action on $G \times G$ (i.e. $(x, y) g=(x, y g))$ as $\gamma$ does. Then $\beta(\delta(g))=\beta\left(\gamma\left(g, 1_{G}\right)\right)=\left(g, 1_{G}\right)$ and thus $\left(\varepsilon \times 1_{G}\right) \beta(\delta(g))=\left(1_{G}, 1_{G}\right)$ and

$$
s=\left(\varepsilon \times 1_{G}\right) \circ \beta\left(-, 1_{G}\right): G \rightarrow G
$$

is a left antipode since

$$
\begin{aligned}
\mu \circ\left(s \times 1_{G}\right) \circ \delta(g)=s(g) g & =\mu \circ\left(\varepsilon \times 1_{G}\right) \circ \beta\left(g, 1_{G}\right) g \\
& =\mu \circ\left(\varepsilon \times 1_{G}\right) \circ \beta(g, g)=1_{G} .
\end{aligned}
$$

Notice that for our proof we need $\beta$ to be a right $G$-morphism. This does not follow if $\gamma$ is only required to be injective, but it does follow if $\gamma$ is required to be bijective.

By a previous observation the existence of a (left) antipode implies that $G$ is a group (then $\left.\beta(g, h)=\left(g, g^{-1} h\right)\right)$.

Similar to the case of Hopf algebras (e.g. $[16,5.5]$ ), the antipode $s: G \rightarrow G$ allows to find the coinvariants of any $G$-bimodule $A$ as the image of

$$
A \stackrel{\varrho^{A}}{\longrightarrow} G \times A \stackrel{s \times 1_{G}}{\longrightarrow} G \times A \stackrel{\varrho_{A}}{\longrightarrow} A .
$$

Writing $\varrho^{A}(a)=(\rho(a), a)$ for some $\rho: A \rightarrow G, a \in A$, we get

$$
A^{c o G}=\{s(\rho(a)) a \mid a \in A\}=\left\{\rho(a)^{-1} a \mid a \in A\right\} .
$$

Moreover, as observed in [19, Theorem 3.1], in this case the canonical map $G \times A^{c o G} \rightarrow A$ is bijective with the inverse map given by

$$
A \rightarrow G \times A^{c o G}, \quad a \mapsto\left(\rho(a), \rho(a)^{-1} a\right) .
$$

This implies that the evaluation map $G \times \operatorname{Map}_{G}^{G}(G, A) \rightarrow A$ is also bijective.

Summarising the information collected above we can transfer characterisations of Hopf algebras to the following

5.20. Characterisations of groups. For a set $G$ and the functor $\bar{G}=G \times-$, the following assertions are equivalent in the category of sets:

(a) The functor $\bar{G}=G \times-$ is a Hopf monad (in the sense 5.15);

(b) $G$ has a group structure;

(c) $G$ is a semigroup and the functor $\bar{G}=G \times-$ is a mixed bimonad with entwining $\psi$ (see above) and one of the following equivalent conditions holds: 
(i) $\bar{G}$ has a (left) antipode;

(ii) the map $\gamma_{G}: G \times G \rightarrow G \times G,(g, h) \mapsto(g, g h)$, is bijective;

(iii) $\phi_{\bar{G}}^{\bar{G}}$ : Set $\rightarrow \operatorname{Set}_{\bar{G}}^{\bar{G}}, X \mapsto G \times X$, is an equivalence;

(iv) $\operatorname{Mor}_{\frac{G}{G}}(G,-): \operatorname{Set}_{\bar{G}}^{\bar{G}} \rightarrow \operatorname{Set} i s$ an equivalence.

Proof. $\quad(b) \Rightarrow(c)$ (ii) is obvious and the equivalence of (a),(b),(c)(i), and (c)(ii) is clear from the preceding comments.

(c) (ii) $\Rightarrow$ (c)(iii) By the given condition in (c), the functor $\phi \frac{\bar{G}}{G}$ : $\operatorname{Set} \rightarrow \operatorname{Set}_{\bar{G}}^{\bar{G}}$ is full and faithful. To prove that it is an equivalence it remains to show that it is representative. This follows from the fact that the evaluation map $G \times \operatorname{Map}_{G}^{G}(G, A) \rightarrow A$ is bijective.

(c)(iii) $\Rightarrow(\mathrm{c})(\mathrm{iv})$ The functor $\operatorname{Mor}_{\bar{G}} \overline{\bar{G}}(G,-)$ is right adjoint to the functor $G \times-$.

(c) (iv) $\Rightarrow($ a) is obvious.

The functor $\operatorname{Map}(C,-)$ for some coalgebra $C$ in Set is also considered in Szlachányi $[48,4.6]$.

\section{$6(F, G)$-dimodules}

In the preceding sections we were concerned with compatibility conditions between algebras and coalgebras. There is also a way to subsume algebras and coalgebras under one notion. This was done by Hagino while studying categorical data types in [25, Definition 3.1.2] where he introduced dialgebras (the name being suggested by Bob McKay, although other meanings for this term exist, e.g. [34]). These are not based on an endofunctor but on two functors between two different categories. In consistency with our previous notation we prefer the name dimodules instead of dialgebras for the basic notion.

6.1. $(\boldsymbol{F}, \boldsymbol{G})$-dimodules. Let $F, G: \mathbb{A} \rightarrow \mathbb{B}$ be two functors between categories $\mathbb{A}$ and $\mathbb{B}$. An $A \in \operatorname{Obj}(\mathbb{A})$ is called an $(F, G)$-dimodule if there is a morphism

$$
\vartheta_{A}: F(A) \rightarrow G(A) \quad \text { in } \mathbb{B} .
$$

A morphism $f: A \rightarrow A^{\prime}$ between two $(F, G)$-dimodules is characterised by commutativity of the diagram

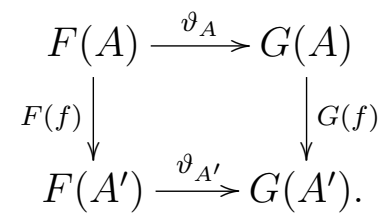


With this morphisms the class of all $(F, G)$-dimodules form a category which we denote by $(F, G)$-Dim.

This is a straightforward extension of the earlier notions of module and comodule: for $\mathbb{A}=\mathbb{B}$ and a functor $F: \mathbb{A} \rightarrow \mathbb{A},(F, I)$-dimodules are $F$ modules, and $(I, F)$-dimodules are $F$-comodules.

Clearly, in case there is a natural transformation $\alpha: F \rightarrow G$, then every $A \in \operatorname{Obj}(\mathbb{A})$ is an $(F, G)$-dimodule by $\alpha_{A}: F(A) \rightarrow G(A)$.

Also, the mixed modules considered in 5.7 are $(F, G)$-dimodules by the composed morphism $F(A) \stackrel{h}{\longrightarrow} A \stackrel{k}{\longrightarrow} G(A)$. Certainly not all dimodules are of this type. An example of an $(F, G)$-dimodule (based on the category of sets) which is neither an $F$-module nor a $G$-comodule is given by Poll and Zwanenberg in [42]. They also investigate how properties of invariants and bisimulations can be generalised from (co-)modules to dimodules.

As outlined in [25, page 50], the great freedom given by the definition allows, for example, to characterise a natural number object in a category $\mathbb{A}$ as an initial object in the category of $(F, G)$-dimodules for the functors

$$
F, G: \mathbb{A} \rightarrow \mathbb{A} \times \mathbb{A}, \quad F(A)=(1, A), G(A)=(A, A), \text { for } A \in \operatorname{Obj}(\mathbb{A}) .
$$

The generality of the notion is also underlined by the fact that it subsumes adjoint pairs of functors as shown in [25, Proposition 3.1.4]:

6.2. $(F, G)$-dimodules and adjoint functors. For categories $\mathbb{A}, \mathbb{B}$, and $\mathbb{D}$, let $T: \mathbb{A} \times \mathbb{B} \rightarrow \mathbb{D}$ and $S: \mathbb{A} \times \mathbb{B}^{o p} \rightarrow \mathbb{D}$ be functors. For $B \in \mathbb{B}$ these induce functors

$$
F_{B}:=T(-, B): \mathbb{A} \rightarrow \mathbb{D} \quad \text { and } \quad G_{B}:=S(-, B): \mathbb{A} \rightarrow \mathbb{D} .
$$

Denote by

$$
\left(\lambda(B), \eta_{B}\right) \text { the initial object, } \quad\left(\rho(B), \gamma_{B}\right) \text { the final object }
$$

in the category $\left(F_{B}, G_{B}\right)$-Dim. Then:

(1) If $\left(\lambda(B), \eta_{B}\right)$ exists for every $B \in \mathbb{B}$, then there is a functor

$$
\lambda(-): \mathbb{B} \rightarrow \mathbb{A}
$$

(2) If $\left(\rho(B), \eta_{B}\right)$ exists for every $B \in \mathbb{B}$, then there is a functor

$$
\rho(-): \mathbb{B}^{o p} \rightarrow \mathbb{A} \text {. }
$$

(3) For a functor $F: \mathbb{A} \rightarrow \mathbb{B}$, the left and right adjoint functors can be derived from suitable functors 


$$
T: \mathbb{A} \times \mathbb{B} \rightarrow \mathbb{B} \text { and } S: \mathbb{A} \times \mathbb{B}^{o p} \rightarrow \mathbb{B} .
$$

As an example for the behaviour with respect to (co)limits in the general case we show:

6.3. Limits of $(F, G)$-dimodules. Let $F, G: \mathbb{A} \rightarrow \mathbb{B}$ be functors between categories. Let $L: \boldsymbol{\Lambda} \rightarrow(F, G)$-Dim be a functor, $\boldsymbol{\Lambda}$ a small category.

(1) If $F$ preserves colimits and $\lim _{\longrightarrow} L$ exists in $\mathbb{A}$, it belongs to $(F, G)$-Dim.

(2) If $G$ preserves limits and $\lim _{\longleftarrow}$ exists in $\mathbb{A}$, it belongs to $(F, G)-\mathrm{Dim}$.

Proof. (1) We have the following diagram in $\mathbb{B}$ :

$$
\begin{gathered}
F(L(\lambda)) \stackrel{F\left(\epsilon_{\lambda}\right)}{\longrightarrow} F(\stackrel{\lim }{\longrightarrow} L) \\
\quad \theta_{L(\lambda)} \\
G(L(\lambda)) \stackrel{G\left(\epsilon_{\lambda}\right)}{\longrightarrow} G(\stackrel{\lim }{\longrightarrow} L),^{\longrightarrow}
\end{gathered}
$$

where the $G\left(\epsilon_{\lambda}\right) \circ \theta_{L(\lambda)}$ form a compatible family of morphisms. By the colimit property there exists $F\left(\lim _{\longrightarrow} L\right) \rightarrow G\left(\lim _{\longrightarrow} L\right)$. Hence $\underset{\lim }{\longrightarrow}$ is an $(F, G)$-module.

(2) This is shown with a similar argument.

For two endofunctors $F$ and $G$, the notion of dimodules subsumes modules and comodules. In this situation one may consider natural transformations between $F F, F G$, etc. and the following definition may be of interest.

6.4. Monadic pairs and their dimodules. Consider two endofunctors $F, G: \mathbb{A} \rightarrow \mathbb{A}$ of any category $\mathbb{A}$. Call $(F, G)$ a monadic pair if there are natural transformations

$$
\nu: F F \rightarrow G F, \quad \eta: G \rightarrow F, \quad \psi: F G \stackrel{F \eta}{\longrightarrow} F F \stackrel{\nu}{\longrightarrow} G F
$$

such that the following diagrams commute:
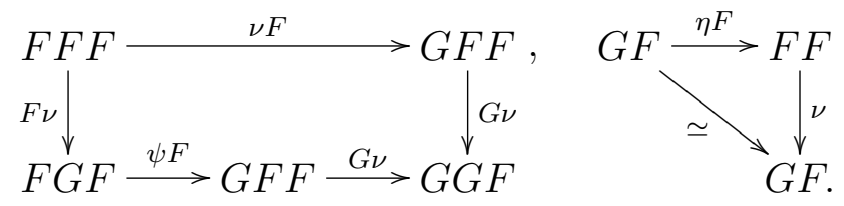

An $A \in \operatorname{Obj}(\mathbb{A})$ is said to be an $(F, G)$-dimodule if there is a morphism

$$
\vartheta_{A}: F(A) \rightarrow G(A)
$$

yielding commutative diagrams
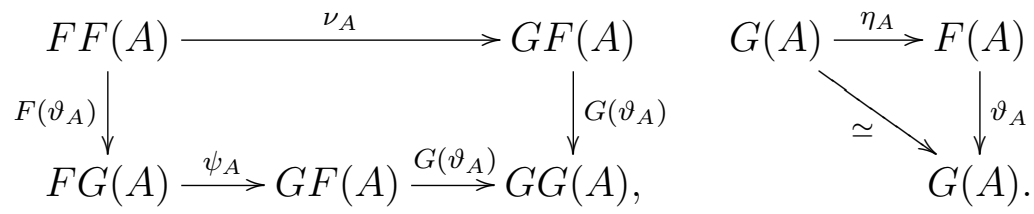
For any monad $F: \mathbb{A} \rightarrow \mathbb{A},(F, I)$ is a monadic pair and $F$-modules are $(F, I)$-dimodules.

For a monadic pair canonical dimodules arise in the following way.

6.5. $F(A)$ and $G(A)$ as $(F, G)$-dimodules. Let $(F, G)$ be a monadic pair of endofunctors of the category $\mathbb{A}$.

(1) For any $A \in \operatorname{Obj}(\mathbb{A}), F(A)$ is a (unital) $(F, G)$-dimodule by

$$
\nu_{A}=\vartheta_{F(A)}: F F(A) \rightarrow G F(A)
$$

(2) If $A$ is an $(F, G)$-dimodule with $\vartheta_{A}: F(A) \rightarrow G(A)$, then $G(A)$ is an $(F, G)$-dimodule by

$$
\vartheta_{G(A)}: F G(A) \stackrel{\psi_{A}}{\longrightarrow} G F(A) \stackrel{G\left(\vartheta_{A}\right)}{\longrightarrow} G G(A)
$$

and $\vartheta_{A}$ is an $(F, G)$-dimodule morphism.

Dual to the previous case one may consider

6.6. Comonadic pairs and their dimodules. A pair of endofunctors $F, G$ : $\mathbb{A} \rightarrow \mathbb{A}$ is said to be a comonadic pair if there are natural transformations

$$
\kappa: F G \rightarrow G G, \quad \varepsilon: G \rightarrow F, \quad \varphi: F G \stackrel{\kappa}{\longrightarrow} G G \stackrel{G \varepsilon}{\longrightarrow} G F
$$

inducing commutative diagrams
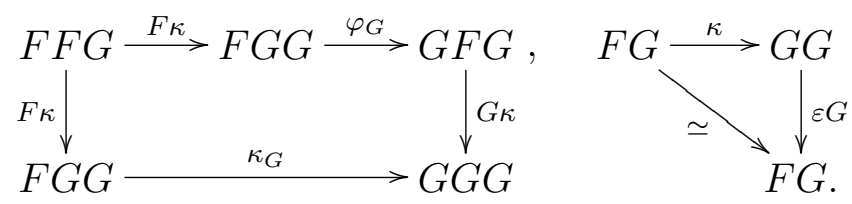

An $A \in \operatorname{Obj}(\mathbb{A})$ is said to be an $(F, G)$-dimodule if there is a morphism

$$
\gamma_{A}: F(A) \rightarrow G(A)
$$

yielding commutative diagrams

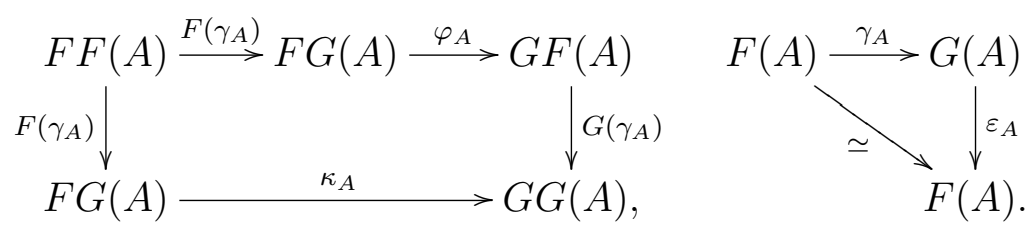

Obviously for any comonad $G: \mathbb{A} \rightarrow \mathbb{A},(I, G)$ is a comonadic pair and $G$-comodules are $(I, G)$-dimodules.

Again we have canonical candidates for dimodules. 
6.7. $F(A)$ and $G(A)$ as $(F, G)$-dimodules. Let $(F, G)$ be a comonadic pair of endofunctors of $\mathbb{A}$.

(1) For any $A \in \mathbb{A}, G(A)$ is a (counital) $(F, G)$-dimodule by

$$
\kappa_{A}=\gamma_{G(A)}: F G(A) \rightarrow G G(A) .
$$

(2) If $A$ is an $(F, G)$-dimodule with

$$
\varrho^{A}: F(A) \rightarrow G(A)
$$

then $F(A)$ is an $(F, G)$-comodule by the morphism

$$
\varrho^{F(A)}=F F(A) \stackrel{F\left(\varrho^{A}\right)}{\longrightarrow} F G(A) \stackrel{\varphi_{A}}{\longrightarrow} G F(A)
$$

and $\varrho^{A}$ is an $(F, G)$-dimodule morphism.

The notions of monadic and comonadic pairs of endofunctors are attempts to generalise monads and comonads. Of course other sets of conditions are possible. Their usefulness will depend on the importance of the class of examples to be found. Notice that natural morphisms of the type $T T \rightarrow T F$ for endofunctors $F, T$ show up, for example, in the study of crossed products in the sense of Brzeziński (see 4.8).

Acknowledgements. The author is grateful to Gabriella Böhm, Claudia Menini, Zoran Škoda, and Bachuki Mesablishvili for their continuous interest and discussions on the subject and for useful comments and hints to relevant literature. He also wants to thank Tomasz Brzeziński for valuable advice and John Clark for his careful proof reading. The referee pointed out some inaccuracies in the previous version and the author is very grateful for this.

\section{References}

[1] Adámek, J. and and Porst, H.E., From varieties of algebras to covarieties of coalgebras, Electronic Notes in Theoretical Computer Science 44.1 (2001)

[2] Baez, J.C., R-commutative geometry and quantization of Poisson algebras, Adv. Math. 95(1), 61-91 (1992)

[3] Barr, M., Composite cotriples and derived functors, in Sem. Triples Categor. Homology Theory, Springer LN Math. 80, 336-356 (1969)

[4] Barr, M. and Wells, Ch., Toposes, triples and theories, Reprints in Theory and Applications of Categories 12, 1-288 (2005) 
[5] Beck, J., Triples, modules and cohomology, Ph.D. thesis Columbia University (1967), republished in: Reprints in Theory and Applications of Categories 2, 1-59 (2003)

[6] Beck, J., Distributive laws, in Seminar on Triples and Categorical Homology Theory, B. Eckmann (ed.), Springer LNM 80, 119-140 (1969)

[7] Bespalov, Y. and Drabant, B., Hopf (bi-)modules and crossed modules in braided monoidal categories, J. Pure Appl. Algebra 123(1-3), 105-129 (1998)

[8] Böhm, G., Nill, F., and Szlachányi, K., Weak Hopf algebras. I., Integral theory and $C^{*}$-structure, J. Algebra 221(2), 385-438 (1999)

[9] Borceux, F., Handbook of categorical algebra. 2: Categories and structures, Encyclopedia of Mathematics and Its Applications 51, Cambridge Univ. Press. (1994)

[10] Borceux, F. and Vitale, E., Azumaya categories, Appl. Categ. Struct. 10(5), 449-467 (2002)

[11] Bruguières, A. and Virelizier, A., Hopf monads, arXiv:math.QA/0604180 (2006)

[12] Brzeziński, T,. Crossed products by a coalgebra, Commun. Algebra 25(11), 3551-3575 (1997)

[13] Brzeziński, T., The structure of corings: induction functors, Maschketype theorem, and Frobenius and Galois-type properties, Algebr. Represent. Theory 5(4), 389-410 (2002)

[14] Brzeziński, T. and Majid, Sh., Comodule bundles, Commun. Math. Phys. 191, No.2, 467-492 (1998)

[15] Brzeziński, T. and Nichita, F.F., Yang-Baxter systems and entwining structures, Commun. Algebra 33(4), 1083-1093 (2005)

[16] Brzeziński, T. and Wisbauer, R., Corings and Comodules, London Math. Soc. Lecture Note Series 309, Cambridge University Press (2003)

[17] Di Luigi, C., Guccione, J.A. and Guccione, J.J., Brzeziński's crossed products and braided Hopf crossed products, Commun. Algebra 32(9), 3563-3580 (2004)

[18] Caenepeel, S., Ion, B., Militaru, G. and Zhu, Shenglin, The factorization problem and the smash biproduct of algebras and coalgebras, Algebr. Represent. Theory 3(1), 19-42 (2000) 
[19] Caenepeel, S. and De Lombaerde, M., A categorical approach to Turaev's Hopf group-coalgebras, Commun. Algebra 34(7), 2631-2657 (2006)

[20] Caenepeel, S., Wang Dingguo and Yin Yanmin, Yetter-Drinfeld modules over weak Hopf algebras and the center construction, Ann. Univ. Ferrara - Sez. VII - Sc. Mat. 51, 69-98 (2005)

[21] Eilenberg, S. and Moore, J.C., Adjoint functors and triples, Ill. J. Math. 9, 381-398 (1965)

[22] Guccione, J.A. and Guccione, J.J., Theory of braided Hopf crossed products, J. Algebra 261(1), 54-101 (2003)

[23] Gumm, H.P., Universelle Coalgebra, in Allgemeine Algebra Ihringer, Th., Berliner Stud. zur Math., Band 10, Heldermann Verlag, 155-207 (2003)

[24] Gumm, H.P., Elements of the general theory of coalgebras, LUATCS'99, Rand Africaans University, Johannesburg, South Africa (1999) (see http://www.mathematik.uni-marburg.de/gumm/)

[25] Hagino, Tatsuya, A categorical programming language, $\mathrm{PhD}$ Thesis, University of Edinburgh (1987)

[26] Hobst, D. and Pareigis, B., Double quantum groups, J. Algebra 242(2), 460-494 (2001)

[27] Johnstone, P.T., Adjoint lifting theorems for categories of modules, Bull. Lond. Math. Soc. 7, 294-297 (1975)

[28] Joyal, A. and Street, R., Braided tensor categories, Adv. Math. 102(1), 20-78 (1993)

[29] Kasangian, S., Lack, S. and Vitale, E.M., Coalgebras, braidings, and distributive laws, Theory Appl. Categ. 13(8), 129-146 (2004)

[30] Kharchenko, V.K., Connected braided Hopf algebras, J. Algebra 307, 24-48 (2007)

[31] Lack, S. and Street, R., The formal theory of monads II, J. Pure Appl. Algebra 175(1-3), 243-265 (2002)

[32] Lawvere, F.W., Functorial Semantics of Algebraic Theories, Proc. Nat. Acad. Sci. 50, 869-872 (1963)

[33] Lenisa, M., Power, J. and Watanabe H., Distributivity for endofunctors, pointed and co-pointed endofunctors, monads and comonads, Elect. Notes in Theor. Comp. Science 33, 230-260 (2000) 
[34] Loday, J.-L., Dialgebras, Loday, J.-L. (ed.) et al., Dialgebras and related operads. Berlin: Springer. Lect. Notes Math. 1763, 7-66 (2001)

[35] Mac Lane, S., Categories for the working mathematician, Grad. Texts in Math., Springer New York (1998)

[36] Majid, S., Algebras and Hopf algebras in braided categories, in Advances in Hopf algebras, Bergen, J. et al (ed.), New York, Marcel Dekker, Lect. Notes Pure Appl. Math. 158, 55-105 (1994)

[37] McCrudden, P., Opmonoidal monads, Theory Appl. Categ. 10, 469-485 (2002)

[38] Menini, C. and Stefan, D., Descent theory and Amitsur cohomology of triples, J. Algebra 266(1), 261-304 (2003)

[39] Mesablishvili, B., Monads of effective descent type and comonadicity, Theory Appl. Categ. 16(1), 1-45, electronic only (2006)

[40] Mesablishvili, B., Entwining structures in monoidal categories, preprint

[41] Moerdijk, I., Monads on tensor categories, J. Pure Appl. Algebra 168(23), 189-208 (2002)

[42] Poll, E. and Zwangenburg, J., From modules and comodules to dimodules, University of Nijmegen, The Netherlands, http://www.cs.kun.nl/ \{erikpoll,janz $\}$

[43] Power, J. and Watanabe, H., Combining a monad and a comonad, Theor. Comput. Sci. 280, No.1-2, 137-162 (2002)

[44] Schauenburg, P., On the braiding on a Hopf algebra in a braided category, New York J. Math. 4, 259-263, electronic only (1998)

[45] Škoda, Z., Distributive laws for actions of monoidal categories, arXiv:math.CT/0406310 (2004)

[46] Škoda, Z., Noncommutative localization in noncommutative geometry, in Noncommutative localization in algebra and topology, Ranicki, A., London Math. Soc. LNS 330, Cambridge University Press (2006)

[47] Street, R., The formal theory of monads, J. Pure Appl. Algebra 2, 149$168(1972)$

[48] Szlachányi, K., The monoidal Eilenberg-Moore construction and bialgebroids, J. Pure Appl. Algebra 182(2-3), 287-315 (2003) 
[49] Takeuchi, M., Survey of braided Hopf algebras, in New trends in Hopf algebra theory, Proc. Coll. Quantum Groups and Hopf Algebras, La Falda, Argentina 1999, Andruskiewitsch, N. et al.(ed.), Providence, RI: American Math. Soc., Contemp. Math. 267, 301-323 (2000)

[50] Tanaka, M., Pseudo-distributive laws and a unified framework for variable binding, PhD Thesis, University of Edinburgh (2005)

[51] Tanaka, M. and Power, J., Pseudo-distributive laws and axiomatics for variable binding, High.-Order Symb. Comput. 19(2-3), 305-337 (2006)

[52] Turi, D. and Plotkin, G., Towards a mathematical operational Semantics, Proceedings 12th Ann. IEEE Symp. on Logic in Computer Science, LICS'97, Warsaw, Poland (1997)

[53] Van Daele, A. and Wang Shuanhong, New braided crossed categories and Drinfeld quantum double for weak Hopf group coalgebras, Commun. Algebra, to appear

[54] van Osdol, D. H., Sheaves in regular categories, in Exact categories and categories of sheaves, Springer Lecture Notes Math. 236, 223-239 (1971)

[55] Wisbauer, R., Weak corings, J. Algebra 245(1), 123-160 (2001)

[56] Wisbauer, R., On Galois comodules, Commun. Algebra 34(7), 2683-2711 (2006)

[57] Wolff, H., $\mathcal{V}$-localizations and $\mathcal{V}$-monads, J. Algebra 24, 405-438 (1973) 